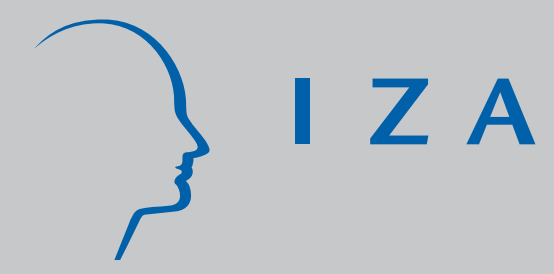

IZADP No. 1672

Exports and Labour Demand:

Searching for Functional Structure in Multi-Output Multi-Skill Technologies

Bertrand M. Koebel

J uly 2005 


\title{
Exports and Labour Demand: Searching for Functional Structure in Multi-Output Multi-Skill Technologies
}

\author{
Bertrand M. Koebel \\ BETA-Thème, Louis Pasteur University, Strasbourg \\ and IZA Bonn
}

Discussion Paper No. 1672

July 2005

IZA

P.O. Box 7240

53072 Bonn

Germany

Phone: +49-228-3894-0

Fax: +49-228-3894-180

Email: iza@iza.org

\begin{abstract}
Any opinions expressed here are those of the author(s) and not those of the institute. Research disseminated by IZA may include views on policy, but the institute itself takes no institutional policy positions.
\end{abstract}

The Institute for the Study of Labor (IZA) in Bonn is a local and virtual international research center and a place of communication between science, politics and business. IZA is an independent nonprofit company supported by Deutsche Post World Net. The center is associated with the University of Bonn and offers a stimulating research environment through its research networks, research support, and visitors and doctoral programs. IZA engages in (i) original and internationally competitive research in all fields of labor economics, (ii) development of policy concepts, and (iii) dissemination of research results and concepts to the interested public.

IZA Discussion Papers often represent preliminary work and are circulated to encourage discussion. Citation of such a paper should account for its provisional character. A revised version may be available directly from the author. 


\section{ABSTRACT}

\section{Exports and Labour Demand: Searching for Functional Structure in Multi-Output Multi-Skill Technologies*}

In order to simplify the representation of a technological relationship between inputs and outputs, a production unit's technology must typically satisfy some restrictive conditions, some of them being well known in the literature. This paper presents new results for aggregating labour inputs and outputs, in terms of restrictions on elasticities of scale and substitution. These conditions are then empirically investigated, in a framework that is flexible and does not lose its flexibility after separability being imposed. The empirical findings of the exact approach to aggregation are found to be rather pessimistic on the possibility to provide a simplified representation.

JEL Classification: $\quad$ C33, D24, E10, J23, L60

Keywords: $\quad$ aggregation, separability, flexibility, exports, labour demand, Box-Cox, system serial correlation

Corresponding author:

Bertrand M. Koebel

BETA-Thème

61 avenue de la Forêt Noire

67085 Strasbourg Cedex

France

Email: koebel@cournot.u-strasbg.fr

\footnotetext{
* I would like to thank Andrew Chesher, Martin Falk, Stefan Hoderlein, Frédéric Jouneau-Sion, François Laisney, Gauthier Lanot and Alban Thomas for most helpful comments, as well as the participants of the seminars held at Innsbruck, London, Louvain-la-Neuve, Mount Sainte-Odile, Strasbourg and Toulouse. I also want to thank two anonymous referees for their advices.

This research has been supported by a Marie Curie Fellowship of the European Community programme Training and Mobility of Researchers under the contract number HPMF-CT-2000-00605.
} 


\section{Introduction}

The theory of input demand is based on aggregate quantities and prices whose existence is often debated. From the work of Leontief [1947] and Gorman [1995] it is clear that the conditions enabling to represent exactly a bunch of goods by a composite commodity are stringent. Whether these requirements are fulfilled or not by production technologies and consumer preferences can be investigated empirically once the corresponding theoretical conditions have been given an observable content. Since Berndt and Christensen [1973], Russell [1975] and Blackorby and Russell [1976], it is well known that inputs and prices can be aggregated within a technology - a structure called homothetic separability - if and only if the elasticities of scale and substitution satisfy some restrictions. As the elasticities can be estimated, the road to empirical investigations is paved, and following Berndt and Christensen [1974] a substantial number of empirical studies have been published.

Two problems related to homothetic separability are addressed in this paper. First, aggregates that are implicitly defined by homothetic separability are nonlinear functions of elementary goods or prices, and do not correspond to the aggregate variables that are provided by statistical offices. Available aggregate quantities and prices are usually additive and/or linearly homogeneous in elementary quantities (or prices), as Laspeyres and Paasche indices for example. They can be consistently used in empirical research only if the production technology is additively and/or homothetically separable. The second problem with homothetic separability goes in the reverse direction. Requiring aggregators to be homothetic implies restrictions on the production technology that are not necessary for an aggregate representation of the technology. Tests can therefore reject homothetic aggregation although an aggregate quantity in fact exists, as highlighted by Blackorby, Schworm and Fisher [1986].

This paper copes with these problems and provides a formal characterization of eight different structures that allow to exactly represent a bundle of inputs (or outputs) by a scalar. These characterizations are then used for empirically investigating the possibility to aggregate three different types of labour inputs and two kinds of outputs. A further contribution of the paper is that it considers both aggregation of inputs that are optimally allocated and aggregation of quasi-fixed outputs. It is shown that different types of flexible labour inputs can be aggregated if and only if these demands react "similarly" to a change in the explanatory variables. Regarding the quasi-fixed outputs, they can be aggregated if and only if they exert a "similar" impact on all input demand functions. These conditions restrict the way the educational structure of labour and the output structure are allowed to be related. If the observed shift in the educational structure of labour is in fact related to the shift in the output structure, results obtained from aggregate models can be suspected of aggregation biases.

The empirical part of the paper investigates the validity of different aggregate representations for German manufacturing industries. Aggregation over three flexible labour inputs (workers with a university degree, those with a vocational degree and those without formal diploma) and over two quasi-fixed outputs (production sold in Germany and exports) is considered. Not only is the share of exports in total production in German manufacturing high - it represented $23 \%$ of total output in 1978 -, its importance also grew over time to reach $31 \%$ in 1994. During this period the educational structure of 
labour inputs also drastically changed. Whereas the number of workers without any formal degree declined by about $46 \%$, the number of workers with a vocational degree increased by $12 \%$ and those with a university degree almost doubled. This paper shows that the question about the validity of aggregates for labour and output is linked to the degree of interrelation between the structure of outputs and labour inputs.

Empirical investigations searching for separable structures, however, face a further important problem first highlighted by Blackorby, Primont and Russell [1977]: the imposition of separable structures leads locally flexible functional forms to lose their flexibility property. In that context, it may happen that separability is rejected, not because it is de facto invalid, but just because the restricted function is not able anymore to approximate an arbitrary separable function. This problem has been tackled by Diewert and Wales [1995], who present a specification that does not lose its local flexibility once homothetic separability is imposed. This approach is adopted here to handle separable, homothetic and additive functional structures.

Starting with a multi-output and multi-labour inputs cost function, I first give necessary and sufficient conditions enabling the outputs and labour inputs to be exactly represented by a scalar aggregate quantity (Section 2). Five different types of output aggregation and three types of labour input aggregation are characterized. As within the cost minimization framework outputs are fixed and labour inputs are flexible, the conditions under which outputs and labour inputs can be aggregated are distinct. The necessary and sufficient conditions for eight types of input and output aggregation are all stated nonparametrically, mainly in terms of elasticities of scale and substitution. These results can then be used for empirical investigation (Section 4). For that purpose, I rely on a Box-Cox specification for the cost function (Section 3), and discuss the outcome of the tests for functional structure in Section 5 .

\section{The structure of multi-output technologies}

After introducing the notations and defining several structures which allow to aggregate goods within a technological relationship, I discuss their implications for input demand adjustments.

\subsection{The technology}

Let $y=\left(y_{d}, y_{x}\right)^{\top}$ be a vector of outputs, where $y_{d}$ and $y_{x}$ denote production sold on the domestic market and exports, respectively. Similarly, the vector $z=\left(\ell^{\top}, v^{\top}\right)^{\top}$ groups all variable inputs: labour inputs denoted by the sub-vector $\ell=\left(\ell_{h}, \ell_{s}, \ell_{u}\right)^{\top}$ and non labour inputs $v=\left(v_{k}, v_{m}\right)^{\top}$, comprising capital and intermediate material respectively. The subscript $h$ stands for high-skilled labour, $s$ for skilled labour, $u$ represents unskilled workers; $v_{k}$ denotes capital and $v_{m}$ intermediate materials. The corresponding price vectors are $w=\left(w_{h}, w_{s}, w_{u}\right)^{\top}$ for wages and $q=\left(q_{k}, q_{m}\right)^{\top}$ for input prices. A time trend $t$ is also included as explanatory variable in the technology for indicating that it may change over time in an a priori unrestricted way.

The technological relationship between inputs and outputs is represented by the trans- 
formation function $f$ such that:

$$
f(z, y, t)=0,
$$

where $f$ is twice continuously differentiable. The technology is strictly quasi-concave in inputs in the sense that the inverse functions in any output $y_{j}$ that are obtained from (1) are (locally) strictly quasi-concave in $(\ell, v)$.

Notice that both outputs $y_{x}$ and $y_{d}$ appear as arguments of the technology. In some cases discussed by Hall [1973] and Kohli [1983], the technology (1) exhibits some additional structure which has interesting implications for the relationship between labour demands and exports. The technology $f$ is said to be non-joint in inputs, when there exist functions $F_{d}$ and $F_{x}$ such that (1) can be equivalently represented by

$$
y_{d}=F_{d}\left(\ell^{d}, v^{d}, t\right) \quad \text { and } \quad y_{x}=F_{x}\left(\ell^{x}, v^{x}, t\right),
$$

with $\ell^{d}+\ell^{x}=\ell$ and $v^{d}+v^{x}=v$. In this case, the domestic and exported outputs are produced independently, using their own inputs $\left(\ell^{d}, v^{d}\right)$ and $\left(\ell^{x}, v^{x}\right)$ respectively, and their own production functions $F_{d}$ and $F_{x}$. There are neither economies nor diseconomies of joint production.

The technology is non-joint in outputs and non-labour inputs when the labour input requirement of a given qualification is independent from any other labour qualifications, or more precisely, when there exist functions $F_{h}, F_{s}$ and $F_{u}$, such that (1) can be equivalently represented by

$$
\begin{aligned}
& \ell_{h}=F_{h}\left(v^{h}, y^{h}, t\right), \\
& \ell_{s}=F_{s}\left(v^{s}, y^{s}, t\right), \\
& \ell_{u}=F_{u}\left(v^{u}, y^{u}, t\right),
\end{aligned}
$$

with $v^{h}+v^{s}+v^{u}=v$ and $y^{h}+y^{s}+y^{u}=y$. This structure arises for example when production is executed in different plants. In the first plant (3), only labour type $h$ is required, and combined with inputs $v^{h}$, it produces the quantities $y^{h}$ of the products with technology $F_{h}$. The second and third plants, (4) and (5), use labour types $s$ and $u$, respectively.

Structures that are more interesting in this paper are those allowing to represent outputs and labour inputs by a scalar aggregate. Those structures are thoroughly discussed in Blackorby, Primont and Russell [1978] and Gorman [1995]. A technology is separable in outputs when there exist some continuously differentiable functions $G_{y}$ and $g_{y}$ such that for all values of the arguments

$$
f\left(\ell, v, y_{d}, y_{x}, t\right)=G_{y}\left(\ell, v, g_{y}\left(y_{d}, y_{x}\right), t\right) .
$$

The real valued aggregator function $g_{y}: \mathbb{R}_{+}^{2} \rightarrow \mathbb{R}_{+}$, which is increasing in both arguments, aggregates both types of outputs into a single composite output, so that $G_{y}$ has one argument less than $f$. When the technology is separable in $y$, the marginal rate of substitution between exports and domestic outputs is independent of any other input.

Similarly, the technology is separable in labour inputs when

$$
f(\ell, v, y, t)=G_{\ell}\left(g_{\ell}\left(\ell_{h}, \ell_{s}, \ell_{u}\right), v, y, t\right),
$$

where the labour input aggregator $g_{\ell}: \mathbb{R}_{+}^{3} \rightarrow \mathbb{R}_{+}$is increasing and continuously differentiable.

Most aggregate measures provided by statistical offices are homogeneous and addi- 
tive in the elementary quantities. For that reason it is useful to specialize the above definitions, and allow the function $g_{y}$ or $g_{\ell}$ to be linearly homogeneous, in which case the technology is said to be homothetically separable in the respective partition. When $g_{y}$ is additive, the technology is additively separable in outputs and

$$
f\left(\ell, v, y_{d}, y_{x}, t\right)=G_{y}\left(\ell, v, g_{x}\left(y_{x}\right)+g_{d}\left(y_{d}\right), t\right) .
$$

Similarly, when $g_{\ell}$ is additive in (7),

$$
f\left(\ell, v, y_{d}, y_{x}, t\right)=G_{\ell}\left(g_{h}\left(\ell_{h}\right)+g_{s}\left(\ell_{s}\right)+g_{u}\left(\ell_{u}\right), v, y, t\right),
$$

and $f$ is said to be additively separable in labour inputs. In (8), exports and domestic outputs measured in "efficient units" $g_{x}\left(y_{x}\right)$ and $g_{d}\left(y_{d}\right)$ are perfectly substitutable. Both the requirements of homogeneity and additivity can be combined to obtain a technology which is homothetically additively separable in $y$ (respectively $\ell$ ). When the functions $g_{x}$ and $g_{d}$ are the identity, exports and domestic outputs are perfectly substitutable; from the technological point of view, both goods are identical.

\subsection{Implications for cost and labour demand functions}

The minimal variable costs for producing a bundle $y$ are given by the cost function

$$
\begin{aligned}
c(w, q, y, t) & =\min _{\ell, v}\left\{w^{\top} \ell+q^{\top} v: f(\ell, v, y, t)=0\right\} \\
& =w^{\top} \ell^{*}(w, q, y, t)+q^{\top} v^{*}(w, q, y, t) .
\end{aligned}
$$

As input demand functions are easily obtained from $c$, the cost function is commonly relied on in empirical analysis. From the definition (10), it is clear that any structure imposed on $f$ will be inherited in some way by the cost function. When production is non-joint in inputs for example, we can write

$$
\begin{aligned}
& c\left(w, q, y_{d}, y_{x}, t\right) \\
= & \min _{\ell^{d}, \ell^{x}, v^{d}, v^{x}}\left\{w^{\top}\left(\ell^{d}+\ell^{x}\right)+q^{\top}\left(v^{d}+v^{x}\right): y_{d}=F_{d}\left(\ell^{d}, v^{d}, t\right) \wedge y_{x}=F_{x}\left(\ell^{x}, v^{x}, t\right)\right\} \\
= & \min _{\ell^{d}, v^{d}}\left\{w^{\top} \ell^{d}+q^{\top} v^{d}: y_{d}=F_{d}\left(\ell^{d}, v^{d}, t\right)\right\}+\min _{\ell^{x}, v^{x}}\left\{w^{\top} \ell^{x}+q^{\top} v^{x}: y_{x}=F_{x}\left(\ell^{x}, v^{x}, t\right)\right\} \\
= & C_{d}\left(w, q, y_{d}, t\right)+C_{x}\left(w, q, y_{x}, t\right) .
\end{aligned}
$$

A simple test for the technology to be nonjoint in inputs consists in checking whether the marginal cost of domestic production is independent of exports:

$$
\frac{\partial^{2} c}{\partial y_{d} \partial y_{x}}=0 .
$$

By Shephard's Lemma, the optimal labour demands obtained under input nonjointness are given by:

$$
\ell_{j}^{*}\left(w, q, y_{d}, y_{x}, t\right)=\ell_{j}^{d}\left(w, q, y_{d}, t\right)+\ell_{j}^{x}\left(w, q, y_{x}, t\right), \quad j \in\{h, s, u\} .
$$

The demand for each input can be seen as the sum of two components: the input demand required for producing domestic goods and the one used for producing exports.

Similarly, it can be shown that the technology is non-joint in outputs and non-labour inputs, if and only if (iff)

$$
c(w, q, y, t)=C_{h}\left(w_{h}, q, y, t\right)+C_{s}\left(w_{s}, q, y, t\right)+C_{u}\left(w_{u}, q, y, t\right),
$$


or equivalently in terms of labour demands,

$$
\ell_{j}^{*}(w, q, y, t)=L_{j}\left(w_{j}, q, y, t\right), \quad j \in\{h, s, u\} .
$$

In that case, there is no substitution between the different labour inputs, that is, the cross price labour elasticities $\varepsilon\left(\ell_{j}^{*}, w_{i}\right)$ are identically zero:

$$
\varepsilon\left(\ell_{j}^{*}, w_{i}\right) \equiv \frac{\partial \ell_{j}^{*}}{\partial w_{i}} \frac{w_{i}}{\ell_{j}^{*}}=0, \quad i, j \in\{h, s, u\}, i \neq j .
$$

It is well known that when the technology is separable in outputs, the cost function can be written as

$$
c\left(w, q, y_{d}, y_{x}, t\right)=B_{y}\left(w, q, g_{y}\left(y_{d}, y_{x}\right), t\right),
$$

where the aggegator $g_{y}$ is the same as in (6). This structure is equivalent to:

$$
\partial\left(\frac{\partial c / \partial y_{d}}{\partial c / \partial y_{x}}\right) / \partial w_{i}=\partial\left(\frac{\partial c / \partial y_{d}}{\partial c / \partial y_{x}}\right) / \partial q_{j}=\partial\left(\frac{\partial c / \partial y_{d}}{\partial c / \partial y_{x}}\right) / \partial t=0, \quad i \in\{h, s, u\}, j \in\{k, m\}
$$

or in terms of elasticities

$$
\begin{aligned}
& \frac{\varepsilon\left(z_{i}^{*}, y_{d}\right)}{\varepsilon\left(c, y_{d}\right)}=\frac{\varepsilon\left(z_{i}^{*}, y_{x}\right)}{\varepsilon\left(c, y_{x}\right)}, \quad i \in\{h, s, u, k, m\}, \\
& \frac{y_{d} \partial^{2} c / \partial t \partial y_{d}}{\varepsilon\left(c, y_{d}\right)}=\frac{y_{x} \partial^{2} c / \partial t \partial y_{x}}{\varepsilon\left(c, y_{x}\right)},
\end{aligned}
$$

Restrictions (17) mean that domestic production and exports have a similar impact on a given input demand, since the (normalized) elasticity of input $z_{i}^{*}$ with respect to domestic output and exports are identical. Besides, the impacts of $y_{d}$ and $y_{x}$ on productivity, measured by $\partial c / \partial t$, must be similar.

The technology is homothetically separable in outputs when, in addition to (17), $g_{y}$ is linearly homogeneous in $y$. The following result, proven in Appendix A, is useful for identifying that structure from the cost function $c$.

Proposition 1. The technology $f$ is homothetically separable in outputs, iff conditions (17) and

$$
\varepsilon\left(\partial c / \partial y_{d}, y_{d}\right)+\varepsilon\left(\partial c / \partial y_{d}, y_{x}\right)=\varepsilon\left(\partial c / \partial y_{x}, y_{d}\right)+\varepsilon\left(\partial c / \partial y_{x}, y_{x}\right)
$$

are satisfied.

This result will be used for testing homothetic separability in outputs. Notice that condition (18) does not restrict the level of overall returns to scale.

In the case where the cost function is additively separable in outputs,

$$
c\left(w, q, y_{d}, y_{x}, t\right)=B_{y}\left(w, q, g_{d}\left(y_{d}\right)+g_{x}\left(y_{x}\right), t\right),
$$

which is satisfied iff in addition to (17), Sono's independence criteria is also fulfilled: ${ }^{2}$

$$
\frac{\partial^{2}\left(\ln \frac{\partial c / \partial y_{d}}{\partial c / \partial y_{x}}\right)}{\partial y_{x} \partial y_{d}}=0
$$

In (19), the function $g_{y}=g_{d}+g_{x}$ is not necessarily homothetic in $y$. As most aggregators available for empirical investigation are both homogeneous and additive, it makes

\footnotetext{
${ }^{2}$ See Blackorby, Primont and Russell [1978, p.159].
} 
also sense to test for whether (17), (18) and (20) are simultaneously satisfied, which corresponds to homothetic additive separability.

Moreover, price and quantity indices commonly published by statistical institutes are weighted sums of elementary prices and quantities. Laspeyres and indices for instance take the form $g_{y}(y)=\delta_{d} y_{d}+\delta_{x} y_{x}$, where $\delta_{d}$ and $\delta_{x}$ are constant weights. This situation can be coined linear separability in outputs.

Proposition 2. The technology $f$ is linearly separable in outputs, iff conditions (17) and

are satisfied.

$$
\varepsilon\left(\partial c / \partial y_{d}, y_{o}\right)=\varepsilon\left(\partial c / \partial y_{x}, y_{o}\right), \quad o=d, x
$$

It is immediate to see that (21) implies (18). Still more restrictive, the requirement

$$
\frac{\partial c}{\partial y_{d}}=\frac{\partial c}{\partial y_{x}},
$$

allows to further simplify the cost function which then becomes: ${ }^{3}$

$$
c\left(w, q, y_{d}, y_{x}, t\right)=B_{y}\left(w, q, y_{d}+y_{x}, t\right) .
$$

In this last case, domestic and exported outputs are strictly identical goods, from a technological point of view. Marginal variations of $y_{d}$ and $y_{x}$ have the same impact on a given input demand. For later reference it is useful to note that although (22) consists only of one equality restriction, it is more stringent than the eight equality restrictions (17) and (21).

For the labour inputs which, contrary to outputs, are optimally adjusted, it is generally assumed that the aggregator $g_{\ell}$ is linearly homogeneous. Under this condition, separability of $f$ in labour inputs is equivalent to separability of the cost function in wages. This point is discussed in detail by Diewert and Wales [1995] for example. Indeed, it is then possible to write

$$
\begin{aligned}
c(w, q, y, t) & =\min _{\ell, v}\left\{w^{\top} \ell+q^{\top} v: G_{\ell}\left(g_{\ell}(\ell), v, y, t\right)=0\right\} \\
& =\min _{\ell}\left\{w^{\top} \ell+A_{\ell}\left(g_{\ell}(\ell), q, y, t\right)\right\} \\
& =\min _{\ell, L}\left\{w^{\top} \ell+A_{\ell}\left(g_{\ell}(\ell), q, y, t\right): g_{\ell}(\ell)=L\right\},
\end{aligned}
$$

where

$$
A_{\ell}\left(g_{\ell}(\ell), q, y, t\right)=\min _{v}\left\{q^{\top} v: G_{\ell}\left(g_{\ell}(\ell), v, y, t\right)=0\right\}
$$

denotes a restricted cost function which is separable in labour inputs. When the labour input aggregator $g_{\ell}$ is linearly homogeneous it is possible to further simplify $c$ :

$$
\begin{aligned}
c(w, q, y, t) & =\min _{\mathbf{L}}\left\{g_{w}(w) \mathbf{L}+A_{\ell}(\mathbf{L}, q, y, t)\right\} \\
& =B_{\ell}(\mathbf{W}, q, y, t),
\end{aligned}
$$

\footnotetext{
${ }^{3}$ To see that (22) implies (23) notice that a cost function satisfying (22) also satisfies (16) and (20), and can therefore be written in the form (19). Then

$$
\frac{\partial c}{\partial y_{d}}=\frac{\partial c}{\partial y_{x}} \Rightarrow \frac{\partial g_{d}}{\partial y_{d}}\left(y_{d}\right)=\frac{\partial g_{x}}{\partial y_{x}}\left(y_{x}\right)=\delta,
$$

where $\delta$ is a constant. Thus $g_{d}\left(y_{d}\right)=\delta y_{d}+\delta_{d}, g_{x}\left(y_{x}\right)=\delta y_{x}+\delta_{x}$ and $g_{d}\left(y_{d}\right)+g_{x}\left(y_{x}\right)=\delta \cdot\left(y_{d}+y_{x}\right)+\delta_{d}+\delta_{x}$. Conversely, (23) also implies (22).
} 
where

$$
\mathbf{W}=g_{w}(w) \equiv \min _{\ell}\left\{w^{\top} \ell: g_{\ell}(\ell)=1\right\}
$$

can be interpreted as an aggregate wage corresponding to one unit of aggregate labour L. The unrestricted cost function $B_{\ell}$ depends only on the aggregate wage level $\mathbf{W}$. Briefly, when the technology is homothetically separable in labour inputs then the cost function $c$ is homothetically separable in labour input prices (and conversely). Testing for homothetic separability can be achieved on the basis of the following restrictions:

$$
\partial\left(\frac{\partial c / \partial w_{i}}{\partial c / \partial w_{j}}\right) / \partial r_{b}=0
$$

for $i, j \in\{h, s, u\}$ and $r_{b} \in\left\{q_{k}, q_{m}, y_{d}, y_{x}, t\right\}$. In terms of elasticities, homothetic separability is equivalent to ${ }^{4}$

$$
\varepsilon\left(\ell_{i}^{*}, r_{b}\right) \equiv \frac{\partial \ell_{i}^{*}}{\partial r_{b}} \frac{r_{b}}{\ell_{i}^{*}}=\frac{\partial \ell_{j}^{*}}{\partial r_{b}} \frac{r_{b}}{\ell_{j}^{*}} \equiv \varepsilon\left(\ell_{j}^{*}, r_{b}\right) .
$$

The elasticities of any distinct labour inputs with respect to a given explanatory variable are identical. Labour demands for different skills must react identically with respect to changes of any of the explanatory variables $q_{k}, q_{m}, y_{d}, y_{x}, t$. There are 10 independent equalities involved in (28).

In most empirical contributions, homotheticity of $g_{\ell}$ is taken for granted. In order to avoid this additional restriction on the function $g_{\ell}$, it is of course possible to rely on the restricted cost function

$$
a(\ell, q, y, t)=\min _{v}\left\{q^{\top} v: f(\ell, v, y, t)=0\right\},
$$

which is equal to $A_{\ell}$ of $(25)$ iff

$$
\partial\left(\frac{\partial a / \partial \ell_{i}}{\partial a / \partial \ell_{j}}\right) / \partial r_{b}=0
$$

for $i, j \in\{h, s, u\}$ and $r_{b} \in\left\{q_{k}, q_{m}, y_{d}, y_{x}, t\right\}$. The following result, proven in Appendix A, gives the corresponding restrictions on the unrestricted labour demand elasticities:

Proposition 3. Under the assumption of cost minimization, the following statements are equivalent:

(i) The technology $f$ is separable in labour inputs,

(ii) The matrix

$$
D_{\ell} \equiv\left[\begin{array}{lll}
\frac{\partial \ell^{*}}{\partial q^{\top}} & \frac{\partial \ell^{*}}{\partial y^{\top}} & \frac{\partial \ell^{*}}{\partial t}
\end{array}\right]
$$

has at most rank one; or equivalently, the matrix

$$
\begin{aligned}
E_{\ell} & \equiv\left[\begin{array}{lllll}
\varepsilon\left(\ell^{*}, q^{\top}\right) & \varepsilon\left(\ell^{*}, y^{\top}\right) & \varepsilon\left(\ell^{*}, t\right)
\end{array}\right] \\
& \equiv\left[\begin{array}{lllll}
\varepsilon\left(\ell_{h}^{*}, q_{k}\right) & \varepsilon\left(\ell_{t}^{*}, q_{m}\right) & \varepsilon\left(\ell_{t}^{*}, y_{d}\right) & \varepsilon\left(\ell_{h}^{*}, y_{x}\right) & \varepsilon\left(\ell_{h}^{*}, t\right) \\
\varepsilon\left(\ell_{s}^{*}, q_{k}\right) & \varepsilon\left(\ell_{s}^{*}, q_{m}\right) & \varepsilon\left(\ell_{s}^{*}, y_{d}\right) & \varepsilon\left(\ell_{s}^{*}, y_{x}\right) & \varepsilon\left(\ell_{s}^{*}, t\right) \\
\varepsilon\left(\ell_{u}^{*}, q_{k}\right) & \varepsilon\left(\ell_{u}^{*}, q_{m}\right) & \varepsilon\left(\ell_{u}^{*}, y_{d}\right) & \varepsilon\left(\ell_{u}^{*}, y_{x}\right) & \varepsilon\left(\ell_{u}^{*}, t\right)
\end{array}\right]
\end{aligned}
$$

has at most rank one.

\footnotetext{
${ }^{4}$ The time "elasticities" are in fact growth rates, defined as

$$
\varepsilon\left(\ell_{i}^{*}, t\right) \equiv \frac{\partial \ell_{i}^{*}}{\partial t} \frac{1}{\ell_{i}^{*}}
$$

They give the percentage change in labour of qualification $i$ as time goes by by 1 unit (a year).
} 
(iii) For any $i, j \in\{h, s, u\}$ and $r_{b} \in\left\{q_{k}, q_{m}, y_{d}, y_{x}, t\right\}$,

$$
\varepsilon\left(\ell_{i}^{*}, r_{b}\right) \sum_{n=1}^{5} \varepsilon\left(\ell_{j}^{*}, r_{n}\right)=\varepsilon\left(\ell_{j}^{*}, r_{b}\right) \sum_{n=1}^{5} \varepsilon\left(\ell_{i}^{*}, r_{n}\right) .
$$

The conditions (31) of Proposition 3 also restrict the way in which prices, output growth and the time trend influence the demands for different qualifications of workers. Like (28), this results imposes some similarity on how different labour demand react to changes in the same explanatory variable. Proposition 3, however, allows to test for separability in labour inputs which are optimally allocated, without assuming homogeneity of the aggregator. It can be seen that this result is implied by homothetic separability in $\ell$ : when the cost function is $B_{\ell}\left(g_{w}(w), q, y, t\right)$, the Hessian matrix with respect to $w$ and $(q, y, t)$ has, indeed, rank one. Point (iii) gives the necessary and sufficient conditions for separability in $\ell$ in terms of the elasticities of scale and substitution. As the sums over $b$ of the left and right hand side of (31) are always equal, there are less (independent) restrictions involved in (31) than in (28). A careful count indicates that 7 out of 15 equalities in (31) are dependent, letting 8 independent restrictions left in condition (iii). The requirements (31) are not only less numerous but also weaker than those (28) prevailing under homothetic separability. Indeed, homogeneity of $g_{\ell}$ in addition implies that $\sum_{n} \varepsilon\left(\ell_{j}^{*}, r_{n}\right)=\sum_{n} \varepsilon\left(\ell_{i}^{*}, r_{n}\right)$, making (31) to coincide with (28) (see Koebel [2001] for details).

Concerning additive separability in labour inputs, the following result is useful for testing that structure:

Proposition 4. The technology $f$ is additively separable in labour inputs iff conditions (31) and

$$
\begin{aligned}
& \varepsilon\left(\ell_{s}^{*} ; w_{u}\right)\left[\varepsilon\left(\ell_{h}^{*} ; w_{s}\right)+\varepsilon\left(\ell_{h}^{*} ; w_{h}\right)\right]=\varepsilon\left(\ell_{h}^{*} ; w_{u}\right)\left[\varepsilon\left(\ell_{s}^{*} ; w_{h}\right)+\varepsilon\left(\ell_{s}^{*} ; w_{s}\right)\right] \\
& \varepsilon\left(\ell_{u}^{*} ; w_{h}\right)\left[\varepsilon\left(\ell_{s}^{*} ; w_{u}\right)+\varepsilon\left(\ell_{s}^{*} ; w_{s}\right)\right]=\varepsilon\left(\ell_{s}^{*} ; w_{h}\right)\left[\varepsilon\left(\ell_{u}^{*} ; w_{s}\right)+\varepsilon\left(\ell_{u}^{*} ; w_{u}\right)\right]
\end{aligned}
$$

are satisfied.

The two requirements (32) of Propositions 4 restrict the admissible values of own and cross price labour demand elasticities. Notice that when labour inputs cannot be substituted for each other, i.e. when all elasticities are equal to zero, (32) is satisfied. A technology which is additively separable in labour input is therefore more general than a technology that is nonjoint in $(y, v)$.

As labour inputs are optimally allocated, it does not make much sense to test whether the different qualifications of labour are identical from a technological point of view. Indeed, such a technology would lead the production unit to use a single labour input: the cheapest one. This is observed at no observation of our sample.

Briefly, from this discussion on functional structure it can be seen that separability conditions imposes two different kinds of restrictions on labour demands. Whereas separability imposes restrictions between the goods belonging to the separable group and those outside, homothetic and additive separability restrict both the relationships within aggregated and between aggregated and non-aggregated goods. 


\section{The empirical model}

A version of Berndt and Khaled's [1979] Box-Cox cost function, extended to nest both the normalized quadratic and the translog functional forms by Koebel, Falk and Laisney [2003] is considered here. Let $p=\left(w^{\top}, q^{\top}\right)^{\top}$ be the price vector. The multiple-output cost function is given by:

$$
c(p, y, t ; \beta, \gamma ; \mu, \theta)=\left\{\begin{array}{cc}
q^{\top} \mu\left(\gamma_{2} C(P, Y, t ; \beta)+1\right)^{1 / \gamma_{2}} & \text { for } \quad \gamma_{2} \neq 0 \\
q^{\top} \mu \exp (C(P, Y, t ; \beta)) & \text { for } \quad \gamma_{2}=0
\end{array}\right.
$$

where, in a first stage,

$$
\begin{aligned}
C(P, Y, t ; \beta)= & \beta_{C}+B_{P} P+B_{Y} Y+\beta_{C t} t \\
& +\frac{1}{2} P^{\top} B_{P P} P+\frac{1}{2} Y^{\top} B_{Y Y} Y+\beta_{t t} t^{2} \\
& +P^{\top} B_{P Y} Y+P^{\top} B_{P t} t+Y^{\top} B_{Y t} t .
\end{aligned}
$$

(This definition of $C$ will be amended later.) Some restrictions are placed on the parameters in order for the Hessian of the cost function to be symmetric in $p$ and $y$ and for parsimony:

$$
\begin{aligned}
\iota_{5}^{\top} B_{P} & =1, \quad B_{P P}=B_{P P}^{\top}, \quad B_{Y Y}=B_{Y Y}^{\top}, \\
\iota_{5}^{\top} B_{P P} & =0, \quad \iota_{5}^{\top} B_{P Y}=\iota_{5}^{\top} B_{P t}=0,
\end{aligned}
$$

where $\iota_{5}$ denotes a $(5 \times 1)$-vector of ones.

The components $P_{i}$ and $Y_{j}$ of the vectors $P$ and $Y$ are Box-Cox like transformations of the corresponding variables $p_{i}$ and $y_{j}:{ }^{5}$

$$
\begin{aligned}
& P_{i}=b_{i}(p)=\left\{\begin{array}{ll}
\frac{\left(p_{i} / q^{\top} \theta\right)^{\gamma_{1}}}{\gamma_{1}} & \text { for } \gamma_{1} \neq 0 \\
\ln \left(p_{i} / q^{\top} \theta\right) & \text { for } \gamma_{1}=0
\end{array}, \quad i=k, h, s, u, m .\right. \\
& Y_{j}=b_{j}\left(y_{j}\right)=\left\{\begin{array}{ccc}
\frac{y_{j}^{\gamma_{1}}}{\gamma_{1}} & \text { for } & \gamma_{1} \neq 0 \\
\ln y_{j} & \text { for } & \gamma_{1}=0
\end{array}, \quad j=d, x .\right.
\end{aligned}
$$

The parameters $\alpha=\left(\beta^{\top}, \gamma^{\top}\right)^{\top}$ have to be estimated. The $(5 \times 1)$-vectors $\mu$ and $\theta$ comprise fixed weights, which are defined below, so that $q^{\top} \mu$ and $q^{\top} \theta$ are kinds of Laspeyres index for non-labour costs. Note that $q^{\top} \theta$ is used to ensure that the functions $b_{i}$ and $C$ are homogeneous of degree zero in prices, and that the multiplicative term $q^{\top} \mu$ appearing in (33) then guarantees the linear homogeneity in prices of $c$.

The two parameters $\gamma_{1}$ and $\gamma_{2}$ capture the way that variables $y_{j}, p_{j}$ and $c$ are changed by the Box-Cox transformations $b_{i}$. For the special case in which $\gamma_{1}=1$ and $\gamma_{2}=1$, the normalized quadratic functional form is obtained, whereas for $\gamma_{1} \rightarrow 0$ and $\gamma_{2} \rightarrow 0$, (33) coincides with the translog. Other usual functional forms that are nested within (33) are discussed by Koebel, Falk and Laisney [2003].

\footnotetext{
${ }^{5}$ With the functionnal form $(34)$ and restrictions $(35)$, both transformations $Y_{j}=y_{j}^{\gamma_{1}} / \gamma_{1}$ and $Y_{j}=\left(y_{j}^{\gamma_{1}}-1\right) / \gamma_{1}$, yield the same cost function.
} 


\section{On the separability-inflexibility debate}

Now that a parametric specification has been chosen, it is interesting to study which restrictions on the parameters of the cost function correspond to the above restrictions on the elasticities of scale and substitution.

A cost function is said to be (locally) flexible when it is able to approximate the level as well as all first and second order derivatives of a twice continuously differentiable cost function at a given point. This concept, first defined by Diewert [1974], initiated numerous contributions in the fields of production and consumer analysis. It is not difficult to see that the Box-Cox specification of this paper, yielding some well known locally flexible specifications as special cases, is itself flexible. Applying Blackorby and Diewert [1979, Theorem 6] allows to conclude that the (unknown) technology dual to the Box-Cox cost function is flexible too.

The main problem with the functional form (33)-(34) is highlighted by Blackorby, Primont and Russell [1977, 1978]: once separability is imposed globally, locally flexible functional forms often lose their flexibility property. In that context, it may happen that separability is rejected, not because it is de facto invalid, but just because the restricted function is not able anymore to approximate an arbitrary separable function.

Consider, for instance, the case of weak separability in outputs for illustrating this drawback. The cost function $c$ is weakly separable in $y$ iff (16) holds, a condition which can be written in the Box-Cox case as: ${ }^{6}$

$$
\begin{aligned}
& \beta_{j x}\left(\beta_{d}+\beta_{d x} Y_{x}+\beta_{d d} Y_{d}+P^{\top} B_{P d}+\beta_{t d} t\right) \\
= & \beta_{j d}\left(\beta_{x}+\beta_{x x} Y_{x}+\beta_{x d} Y_{d}+P^{\top} B_{P x}+\beta_{t x} t\right),
\end{aligned}
$$

for $j=h, s, u, k, m, t$. These equalities are satisfied iff

$$
\begin{aligned}
\beta_{d} \beta_{j x}-\beta_{x} \beta_{j d} & =0 \\
\beta_{j d} \beta_{i x}-\beta_{j x} \beta_{i d} & =0,
\end{aligned}
$$

for $j \in\{h, s, u, k, m, t\}$ and $i \in\{h, s, u, k, m, t, d, x\}$. When for any $j \in\{h, s, u, k, m, t\}$, the equalities

$$
\beta_{j x}=\beta_{j d}=0,
$$

which imply that (39) are fulfilled, then $C$ becomes

$$
\begin{aligned}
\widetilde{C}(P, \mathbf{Y}, t ; \beta)= & \beta_{C}+B_{P} P+\boldsymbol{\beta}_{\mathbf{Y}} \mathbf{Y}+\beta_{t} t \\
& +\frac{1}{2} P^{\top} B_{P P} P+\beta_{t t} t^{2}+P^{\top} B_{P t} t
\end{aligned}
$$

with

$$
\boldsymbol{\beta}_{\mathbf{Y}} \mathbf{Y}=B_{Y} Y+\frac{1}{2} Y^{\top} B_{Y Y} Y
$$

\footnotetext{
$\frac{\partial c / \partial y_{x}}{\partial c / \partial y_{d}}=\frac{\partial C / \partial y_{x}}{\partial C / \partial y_{d}}=\frac{\beta_{x}+\beta_{x x} Y_{x}+\beta_{d x} Y_{d}+P^{\top} A_{P x}+\beta_{t x} t}{\beta_{d}+\beta_{d x} Y_{x}+\beta_{d d} Y_{d}+P^{\top} A_{P d}+\beta_{t d} t} \frac{\partial Y_{x} / \partial y_{x}}{\partial Y_{d} / \partial y_{d}}$$$
\partial\left(\frac{\partial c / \partial y_{x}}{\partial c / \partial y_{d}}\right) / \partial j=0 \Leftrightarrow \partial\left(\frac{\partial C / \partial y_{x}}{\partial C / \partial y_{d}}\right) / \partial j=0 \Leftrightarrow \beta_{j x} \frac{\partial C}{\partial Y_{d}}=\frac{\partial C}{\partial Y_{x}} \beta_{j d}
$$

or equivalently

$$
\begin{aligned}
& \beta_{j x}\left(\beta_{d}+\beta_{d x} Y_{x}+\beta_{d d} Y_{d}+P^{\top} B_{P d}+\beta_{d d} t\right)=\beta_{j d}\left(\beta_{x}+\beta_{x x} Y_{x}+\beta_{x d} Y_{d}+P^{\top} B_{P x}+\beta_{x t} t\right) \\
& \Leftrightarrow \beta_{j x} \beta_{d}-\beta_{j d} \beta_{x}+\left(\beta_{j x} \beta_{d x}-\beta_{j d} \beta_{x x}\right) Y_{x}+\left(\beta_{j x} \beta_{d d}-\beta_{j d} \beta_{d x}\right) Y_{d} \\
& +P^{\top}\left(\beta_{j x} B_{P d}-\beta_{j d} B_{P x}\right)+\left(\beta_{j x} \beta_{d t}-\beta_{j d} \beta_{x t}\right) t=0
\end{aligned}
$$
}


The boldface characters are used to denote aggregate variables and aggregate parameters. The specification (41) is no longer flexible in $(P, \mathbf{Y}, t)$ : the first derivative of $\widetilde{C}$ with respect to $P$ for instance is independent of $Y$. As a consequence, the imposition of global separability conditions on local flexible specifications destroys flexibility. See Blackorby, Primont and Russell [1978, Section 8.3] for a thorough discussion.

In order to avoid the inflexibility drawback, Diewert and Wales [1995] propose a functional specification that comprises more parameters than (33), and that is still locally flexible after imposition of global separability (that is separability over the whole set of possible values of $(p, y, t))$. In the context of output aggregation, Diewert and Wales' strategy would lead to specify $C$ as

$$
\begin{aligned}
C_{\mathbf{Y}}(P, Y, \mathbf{Y}, t ; \beta)= & \beta_{C}+B_{P} P+\beta_{t} t \\
& +\frac{1}{2} P^{\top} B_{P P} P+\beta_{t t} t^{2}+P^{\top} B_{P t} t \\
& +\boldsymbol{\beta}_{\mathbf{Y}} \mathbf{Y}+\frac{1}{2} \boldsymbol{\beta}_{\mathbf{Y Y}} \mathbf{Y}^{2}+P^{\top} \mathbf{B}_{P \mathbf{Y}} \mathbf{Y}+\boldsymbol{\beta}_{\mathbf{Y} t} \mathbf{Y} t \\
& +P^{\top} B_{P Y} Y+Y^{\top} B_{Y t} t,
\end{aligned}
$$

where $\mathbf{Y}$ is defined as in (42). The first three lines of $C_{\mathbf{Y}}$ correspond to a function of $(P, \mathbf{Y}, t)$ that is both flexible in $(P, \mathbf{Y}, t)$ and separable in $y$, whereas the last line is added for allowing $C_{\mathbf{Y}}$ to be non-separable in $y$. When this last line vanishes, the cost function is clearly separable in $y$ (but not conversely as it will be seen below).

As aggregation of output and labour inputs is of interest here, the following specification is considered in the sequel instead of (43), with $P=\left(W^{\top}, Q^{\top}\right)^{\top}$ :

$$
\begin{aligned}
C_{\mathbf{W Y}}(P, Y, \mathbf{W}, \mathbf{Y}, t ; \beta)= & \beta_{C}+B_{Q} Q+\beta_{t} t+\frac{1}{2} Q^{\top} B_{Q Q} Q+Q^{\top} B_{Q t} t+\frac{1}{2} \beta_{t t} t^{2} \\
& +\boldsymbol{\beta}_{\mathbf{Y}} \mathbf{Y}+\frac{1}{2} \boldsymbol{\beta}_{\mathbf{Y Y}} \mathbf{Y}^{2}+Q^{\top} \mathbf{B}_{Q \mathbf{Y}} \mathbf{Y}+\boldsymbol{\beta}_{\mathbf{Y} t} \mathbf{Y} t \\
& +\boldsymbol{\beta}_{\mathbf{W}} \mathbf{W}+\frac{1}{2} \boldsymbol{\beta}_{\mathbf{W} \mathbf{W}} \mathbf{W}^{2}+\mathbf{W B}_{\mathbf{W} Q} Q+\boldsymbol{\beta}_{\mathbf{W} t} \mathbf{W} t+\boldsymbol{\beta}_{\mathbf{W Y}} \mathbf{W Y} \\
& +\mathbf{W B}_{\mathbf{W} Y} Y+Q^{\top} B_{Q Y} Y+Y^{\top} B_{Y t} t \\
& +W^{\top} \mathbf{B}_{W \mathbf{Y}} \mathbf{Y}+W^{\top} B_{W Y} Y+W^{\top} B_{W Q} Q+W^{\top} B_{W t} t
\end{aligned}
$$

The aggregate output $\mathbf{Y} \in \mathbb{R}$ is defined in (42) and the aggregate wage $\mathbf{W} \in \mathbb{R}$ is given by:

$$
\boldsymbol{\beta}_{\mathbf{W}} \mathbf{W}=B_{W} W+\frac{1}{2} W^{\top} B_{W W} W .
$$

For the purpose of identification, the parameters $\boldsymbol{\beta}_{\mathbf{Y}}$ and $\boldsymbol{\beta}_{\mathbf{W}}$ can be normalized:

$$
\boldsymbol{\beta}_{\mathbf{Y}}=\boldsymbol{\beta}_{\mathbf{W}}=1 \text {. }
$$

Some other parameter restrictions are imposed for the sake of parsimony: (35) and $\iota_{5}^{\top} \mathbf{B}_{P \mathbf{Y}}=0$. In the three first lines of (44) the variables $W$ and $Y$ are aggregated into $\mathbf{W}$ and $\mathbf{Y}$. Since this expression is quadratic in $(Q, \mathbf{W}, \mathbf{Y}, t)$, it follows that $C_{\mathbf{W Y}}$ is locally flexible in these variables. The two last lines of (44) introduce the additional disaggregate variables $Y$ and $W$ and thereby they allow the cost function to be nonseparable in $y$ and $w$.

Specification (44) is interesting as it nests specifications (34), (41) and (43). Notice that when $B_{\mathbf{W Y}}=0, \mathbf{B}_{Q \mathbf{Y}}=0, \boldsymbol{\beta}_{\mathbf{Y} t}=\boldsymbol{\beta}_{\mathbf{W Y}}=0$, the cost function is separable in $y$ under 
the usual conditions (39). Furthermore, the condition

$$
\mathbf{B}_{\mathbf{W} Y}=0, B_{Q Y}=0, B_{Y t}=0, B_{W Y}=0,
$$

implies separability in $y$, but is not equivalent to it. ${ }^{7}$ For this reason $y$-separability tests relying on (47), like those formulated by Diewert and Wales [1995], are prone to reject the null hypothesis too often. Similarly,

$$
\mathbf{B}_{W \mathbf{Y}}=B_{W t}=0, B_{W Y}=B_{W Q}=0,
$$

implies global separability in $w$, but not conversely.

In theory, it is possible to give the necessary and sufficient parametric conditions for a technology to be globally separable in output and homothetically separable in labour inputs. These restrictions would be more stringent than the restrictions (17) and (28) which are of local nature. However, the derivation of these parametric conditions is messy and devoid of economic meaning. This observation gives some value added to the characterization in terms of elasticities provided in Section 2, whose validity does not depend upon the parametric specification of the cost function.

The interesting result relative to the specification (33), (44) in comparison to (33)-(34) follows from Diewert and Wales [1995]:

Proposition 5: The cost function $c$ defined by (33), (35), (42), (44) and (45) is

(i) flexible in $(w, q, y, t)$,

(ii) flexible in $(w, q, \mathbf{y}, t)$ when $c$ is globally separable in $y$,

(iii) flexible in $(\mathbf{w}, q, y, t)$ when $c$ is globally separable in $w$,

(iv) flexible in $(\mathbf{w}, q, \mathbf{y}, t)$ when $c$ is globally separable in $w$ and in $y$.

Proposition 5 means that the cost function is flexible in $(w, q, y, t)$ and that this flexibility property is still satisfied when the technology is globally separable in outputs (points ii and iv) and/or homothetically separable in labour inputs (points iii and iv). See Appendix A for a proof. Proposition 5 justifies the use of (44) for testing separability in outputs and/or homothetic separability in labour inputs globally, without losing flexibility. This result will be used in the empirical part of the paper to see whether the loss of flexibility of (33)-(34), once separability is imposed, can explain why separability is often rejected.

\section{Data and regression}

The data used for the empirical investigation are two digit industry data for West German manufacturing, they are available for $N=24$ industries and cover the period from 1976 to $1994(T=19)$. The subscripts $n=1, \ldots, N$ and $t=1, \ldots, T$ denote industry and time respectively. Over this period, total manufacturing exports in constant prices grew at an average annual rate of 3.3 percent, whereas domestic production grew only by 1.1 percent. Turning to the labour inputs, the employment of university graduates, $\ell_{h}$, (measured as full-time equivalents) increased at an average annual rate of 3.8 percent. In contrast, the number of unskilled workers $\ell_{u}$ decreased by 3.3 percent per year. The number of skilled workers grew by 0.6 percent in average. Relative wages do not appear to have changed much during the period. In the aggregate (over all industries and years

\footnotetext{
${ }^{7}$ A weaker set of sufficient conditions for output separability comprises $B \mathbf{W} Y=0$ and (40).
} 
considered in this paper), exports represented roughly 27 percent of the total level of production. Moreover the share of exports in production increased over time: from 23 percent in 1976 to 31 percent in 1994.

To account for heterogeneous technologies we allow some parameters $\beta_{C}$ and $B_{P}$ to be industry-specific. In former work on similar data, Koebel, Falk and Laisney [2003] found that the data transformation parameters $\gamma$ also vary across groups of industries, so that I allow these parameters to vary across industries (hence the notation $\gamma_{1 n}$ and $\left.\gamma_{2 n}\right)$. This means that the functional form of the cost function is allowed to differ across the industries: it may for example be translog for some industries (those with $\gamma_{1 n} \rightarrow 0$ and $\left.\gamma_{2 n} \rightarrow 0\right)$, whereas for others, a normalized quadratic specification $\left(\gamma_{1 n}=\gamma_{2 n}=1\right)$ could be more adequate.

The optimal demand functions $z^{*}$ are related to the cost function by Shephard's Lemma, which is applied here to form the input-output coefficients considered in the regression:

$$
z_{n t} / \mathbf{y}_{n t}^{+}=z_{n t}^{*} / \mathbf{y}_{n t}^{+}+v_{n t} .
$$

For rendering the assumption of homoscedastic residuals more plausible, I consider the input-output ratio $z_{n t} / \mathbf{y}_{n t}^{+}$(total output being given by $\mathbf{y}_{n t}^{+} \equiv y_{d, n t}+y_{x, n t}$ ), instead of the absolute demand levels $z_{n t}$ as regressands.

In the specification (33), the parameters $\theta$ and $\mu$ could be estimated. It can be seen by adapting Diewert and Wales' [1987] argumentation, that the flexibility of the above specification does not depend upon a particular choice for $\theta$. For that reason they have often been fixed, in order to limit the overall number of parameters. These weights are usually defined as a function of inputs and costs, a practice which introduces some correlation between $\theta$ and the residual term $v_{n t}$. To avoid this difficulty, I specify

$$
\theta_{n}=\left(\begin{array}{c}
\theta_{k, n} \\
\theta_{m, n}
\end{array}\right) \equiv \frac{\mu_{n}}{\frac{1}{N-1} \sum_{i \neq n} q_{i, 91}^{\top} v_{i, 91}},
$$

with $\mu_{n}=\left(\mu_{k, n}, \mu_{m, n}\right)^{\top} \equiv \frac{1}{N-1} \sum_{i \neq n} v_{i, 91}$. As all prices are normalized to 1 in 1991, it follows that $q_{n, 91}^{\top} \theta_{n}=1$.

The residual vector $v_{n t}(J \times 1)$ is assumed to be independent of the regressors. It also satisfies $\mathrm{E}\left[v_{n t}\right]=0, \mathrm{E}\left[v_{n t} v_{n t}^{\top}\right]=\Psi$ and may also be subject to first order serial correlation:

$$
v_{n t}=R v_{n, t-1}+\zeta_{n t}, \quad \lim _{j \rightarrow+\infty} R^{j}=0
$$

with $\mathrm{E}\left[\zeta_{n t}\right]=0, \mathrm{E}\left[v_{n, t-1} \zeta_{n t}^{\top}\right]=0, \mathrm{E}\left[\zeta_{n t} \zeta_{n t}^{\top}\right]=\Psi-R \Psi R^{\top}$ and $\mathrm{E}\left[\zeta_{n t} \zeta_{i s}^{\top}\right]=0$ for all $n \neq i$ or $t \neq s$.

The variance matrix of $v(J T N \times 1)$ is $V\left[v v^{\top}\right]=I_{N} \otimes \Phi$, where $\Phi(J T \times J T)$ is given by

$$
\Phi=\left(\begin{array}{cccc}
\Psi & \Psi R^{\top} & \ldots & \Psi R^{\top T-1} \\
R \Psi & \Psi & \cdots & \Psi R^{\top T-2} \\
\vdots & \vdots & & \vdots \\
R^{T-1} \Psi & R^{T-2} \Psi & \ldots & \Psi
\end{array}\right) .
$$

When $R=0$, one obtains the conventional $S U R$ variance matrix $I_{N T} \otimes \Psi$. Preliminary estimates of the conventional $S U R$ residuals were used to build a consistent estimate $\widehat{\Phi}$ of $\Phi$ by equaling the elements of $\Phi$ to their sample analogue. In all cases the matrix $\widehat{\Phi}$ 
was found to be positive definite. As shown by Koebel [2004], this ensures that $\widehat{R}$ is a convergent matrix (in the sense that $\lim _{j \rightarrow+\infty} \widehat{R}^{j}=0$ ).

\section{Empirical results}

\section{1 $\quad$ First model}

The first model considered consists of (49) which is obtained from (33)-(37). The complete model comprises 151 free $\alpha_{n}$-parameters (among which $5 \times 24=120$ industry specific parameters) and $2 \times 24=48 \gamma_{n}$-parameters which have to be estimated on the basis of $5 \times 19 \times 24=2280$ observations. As there are more than 10 observations per parameter, the incidental parameter problem should not be problematic here. This specification of the demand functions does not only include the usual additive fixed effects, but comprises also industry-specific Box-Cox parameters, so that the marginal impacts of output on input demands are by no way restricted to be identical from one industry to the other. This extension is compatible with heterogeneous outputs across industries, contrary to models which are linear in output.

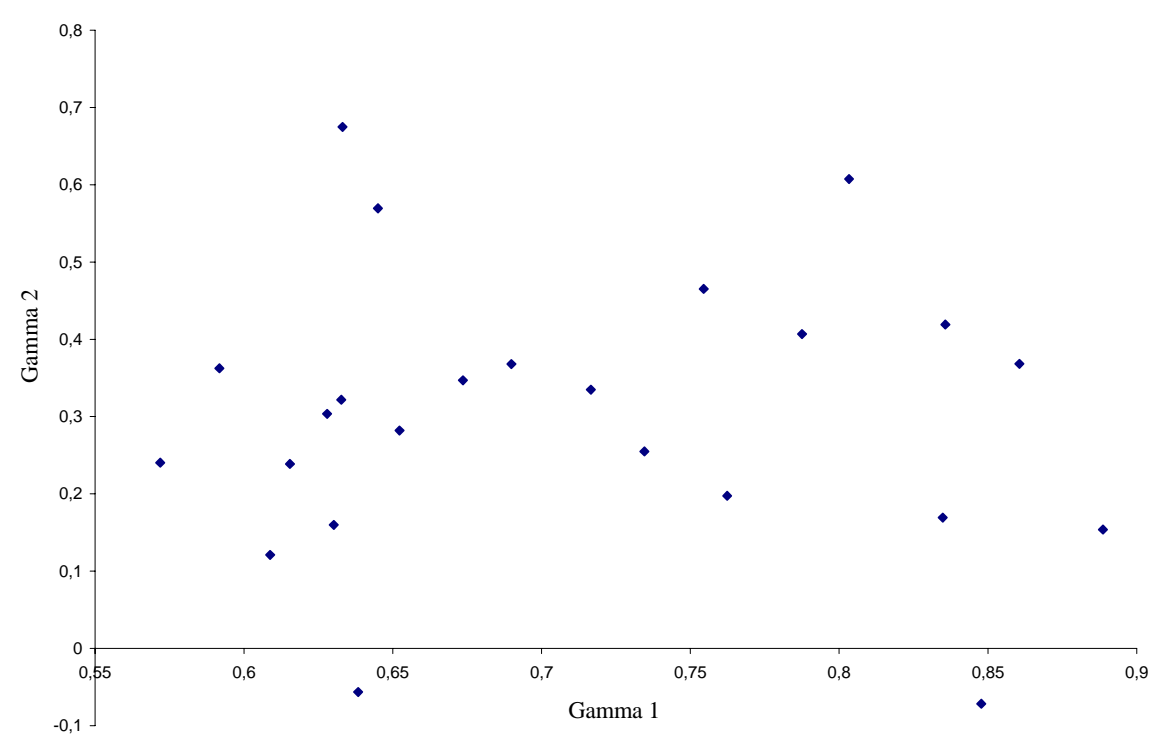

Figure 1: Scatter plot of $\widehat{\gamma}_{1 n}$ and $\widehat{\gamma}_{2 n}$

The estimates of the variable transforming parameters $\gamma_{n}$ are plotted on Figure 1 . In most cases the estimates are included between zero and one: there are only two values for $\widehat{\gamma}_{2 n}$ that lay outside the zero to one interval. All estimates are significantly different from zero. It can be seen on Figure 1, that the estimates of $\widehat{\gamma}_{2 n}$ are smaller (and less dispersed) than those of $\widehat{\gamma}_{1 n}$. The assumption that $\gamma_{1 n}=\gamma_{2 n}$, leading to a Berndt and Khaled [1979] type of specification is rejected by the data. Although there is a cloud of points around the mean point $\bar{\gamma}=(0.71,0.30)$, a test for the hypothesis that the functional family is the same across industries, is rejected at the one percent threshold. Several usual functional specifications which are nested within the Box-Cox 
are all rejected, see also Koebel, Falk and Laisney [2003].

The data were found to exhibit strong serial correlation. Only one estimate of the five eigenvalues of matrix $R$ was real valued. In all cases, however, the modulus of the estimated eigenvalues are below one (between 0.45 and 0.80 ). Thus, a necessary condition for the matrix $\widehat{\Phi}$ to be positive definite is satisfied, and it turned out in fact that $\widehat{\Phi}$ was definite positive.

The estimated values of the elasticities of substitution and scale are reported in Table 1. The own-price elasticities appear on the main diagonal of the upper panel and are all negative. The off diagonal entries correspond to the cross price elasticities and indicate that high-skilled and skilled labour are substitutes as well as skilled and unskilled labour. High-skilled and unskilled labour, however, are complements. It can be seen from Table 1 that the prices $q_{k}$ and $q_{m}$ have a quite different impact on the different qualifications of labour. It turns out that the more skilled is labour, the more it is complementary with capital, $\epsilon\left(\ell_{h}^{*}, q_{k}\right) \leq \epsilon\left(\ell_{s}^{*}, q_{k}\right) \leq \epsilon\left(\ell_{u}^{*}, q_{k}\right) \leq 0$.

The scale elasticities are reported on the lower panel of Table 1. Although all inputs are non-decreasing in $y_{d}$ and in $y_{x}$, the impacts of domestic and exported production on inputs are quite different. With regard to labour inputs, it can be seen that exports are intensive in education, in the sense that $\epsilon\left(\ell_{h}^{*}, y_{x}\right) \geq \epsilon\left(\ell_{s}^{*}, y_{x}\right) \geq \epsilon\left(\ell_{u}^{*}, y_{x}\right)$. The increase in exports in Germany provides thereby a partial explanation for the observed shift in the structure of labour demand in favour of more educated workers. The last line of Table 1 gives the impact of the time trend on input demands. The impact of $t$ on heterogeneous labour inputs is also quite different. Notice that these strong dissimilarities in input demand reactions to changes in $q_{k}, q_{m}, y_{d}, y_{x}$ and $t$, are inconsistent with the conditions favorable to labour aggregation which require that the impact of the different explanatory variables are "similar" across educational levels. Thus, these empirical results foreshadow the difficulties of aggregating labour inputs.

There is also evidence that domestic and exported production have different impacts on a given input: in all cases, $\epsilon\left(x_{j}^{*}, y_{d}\right) \geq \epsilon\left(x_{j}^{*}, y_{x}\right)$ in Table 1 . This observation does not necessarily stand in contradiction with output aggregation: when $c\left(w, q, y_{d}, y_{x}, t\right)=$ $B_{y}\left(w, q, y_{d}+y_{x}, t\right)$, for instance, it follows that $\epsilon\left(x_{j}^{*}, y_{d}\right) \geq \epsilon\left(x_{j}^{*}, y_{x}\right)$ as soon as $y_{d} \geq y_{x}$, although outputs are perfectly identical. In order to study the possibilities of aggregating outputs, the more formal hypotheses, given in Section 2, have to be tested.

Results of the tests for functional structure are summarized in Table 2. The different hypothesis tested are given in the first column. The second column recapitulates the formal expressions of the corresponding equality restrictions which have been presented in Section 2. Column 3 gives the number of independent restrictions that are tested at a given observation point $(n, t)$. As the test statistics depend on the observations $\left(w, q, y_{d}, y_{x}, t\right)$, they take different values over the sample. To avoid dependence upon a arbitrarily chosen reference point, I compute the hypothesis test for each observation point in turn and report both the mean value of the statistic and the percentage of significant violations in columns 4 and 5 of Table 2 .

The main result is that all types of restrictions are strongly rejected. Separability in outputs is rejected for 84.9 and separability in labour input is rejected for 95.4 percent of the observations. The rejection rate increases for the more restrictive versions of homothetic and additive separability. For labour inputs, homothetic separability is 
Table 1: Elasticities of the disaggregate model ${ }^{(1)}$

\begin{tabular}{ccccccc}
\hline \hline$\epsilon\left(j^{*}, i\right)$ & \multicolumn{1}{c}{$\ell_{h}$} & $\ell_{s}$ & $\ell_{u}$ & $v_{k}$ & $v_{m}$ \\
\hline$w_{h}$ & -0.07 & 0.03 & -0.10 & -0.03 & 0.01 \\
& $(-0.8)$ & $(3.6)$ & $(-5.3)$ & $(-4.6)$ & $(6.2)$ \\
$w_{s}$ & 0.41 & -0.34 & 0.20 & -0.08 & 0.05 \\
& $(2.4)$ & $(-10.5)$ & $(6.0)$ & $(-3.0)$ & $(6.3)$ \\
& $w_{u}$ & -0.88 & 0.13 & -0.42 & -0.02 & 0.05 \\
& $(-4.8)$ & $(4.3)$ & $(-6.2)$ & $(-0.8)$ & $(4.5)$ \\
\cline { 3 - 5 }$q_{k}$ & -0.26 & -0.05 & -0.02 & -0.18 & 0.04 \\
& $(-4.9)$ & $(-3.6)$ & $(-1.9)$ & $(-5.2)$ & $(6.7)$ \\
$q_{m}$ & 0.72 & 0.24 & 0.36 & 0.30 & -0.16 \\
& $(7.6)$ & $(6.3)$ & $(6.3)$ & $(9.2)$ & $(-5.3)$ \\
\hline \multirow{2}{*}{$y_{d}$} & 0.80 & 0.54 & 0.60 & 0.58 & 0.70 \\
& $(9.5)$ & $(12.7)$ & $(9.7)$ & $(6.1)$ & $(23.8)$ \\
$y_{x}$ & 0.41 & 0.06 & -0.01 & 0.14 & 0.18 \\
& $(9.0)$ & $(4.3)$ & $(-0.3)$ & $(5.4)$ & $(13.6)$ \\
$t$ & 0.035 & 0.003 & -0.031 & 0.001 & 0.003 \\
& $(8.8)$ & $(3.5)$ & $(-6.6)$ & $(0.4)$ & $(4.5)$ \\
\hline \hline
\end{tabular}

(1) Median value of the elasticities evaluated at the 1985 data and estimated t-values in parentheses.

Table 2: Tests for functional structure, Model 1

\begin{tabular}{|c|c|c|c|c|}
\hline & $\begin{array}{l}\text { Tested } \\
\text { restrictions }\end{array}$ & $\begin{array}{l}\text { Independent } \\
\text { restrictions }\end{array}$ & $\begin{array}{l}\text { Mean } \\
\text { Wald test } \\
(1)\end{array}$ & $\begin{array}{l}\% \text { obs. with } \\
\mathrm{H}_{0} \text { rejected }^{(2)}\end{array}$ \\
\hline \multicolumn{5}{|l|}{ Outputs: } \\
\hline non-jointness & $(12)$ & 1 & $31.8(20.4)$ & 86.0 \\
\hline separability & (17) & 6 & $41.2(17.1)$ & 84.9 \\
\hline homothetic separability & $(17),(18)$ & 7 & $43.1(15.1)$ & 90.1 \\
\hline additive separability & $(17),(20)$ & 7 & $43.4(13.6)$ & 93.0 \\
\hline hom. add. separability & $(17),(21)$ & 8 & $112.6(69.7)$ & 90.5 \\
\hline identical outputs & $(22)$ & 1 & $14.9(14.7)$ & 62.5 \\
\hline \multicolumn{5}{|l|}{ Labour inputs: } \\
\hline non-jointness & (14) & 3 & $59.5(23.3)$ & 100 \\
\hline separability & $(31)$ & 8 & $51.0(22.6)$ & 95.4 \\
\hline homothetic separability & $(28)$ & 10 & $374.5(180.0)$ & 100 \\
\hline additive separability & $(31),(32)$ & 10 & $54.8(20.4)$ & 96.9 \\
\hline
\end{tabular}


rejected for all 456 observation points. This is a logical consequence from the fact that the impacts of prices, outputs and time are very different across the three types of labour inputs (Table 1). Comparable separability structures are more strongly rejected when involving labour inputs as it is the case with respect to output.

Notice that the outcomes of the tests are sometimes inconsistent with the theory: whereas the null of identical outputs is rejected for 62.5 percent of the observations, output separability is invalidated in 84.9 percent of the cases. This finding is paradoxical because output identity implies output separability, but not conversely. This contradiction might be due to the fact that the weaker assumption of separability involves 6 equality restrictions (17), whereas the stronger requirement of output identity only involves one restriction (22). This paradox is related to the lack of invariance of the Wald test to nonlinear transformations. Similarly, the assumption of additive separability is rejected for more observation points that the more stringent homothetic additive separability hypothesis.

The validity of the null assumptions has also been tested globally. This is not a straightforward task, because the test statistics which depend upon observations and estimated parameters, take different values over the sample. The details of the procedure used to test the null globally are given in Appendix B. The different types of restrictions are globally rejected at any reasonable threshold.

For the cases of separability in outputs and homothetic separability in labour inputs, some parametric restrictions implying global separability are known (see (39)). The Wald statistics corresponding to these parametric hypotheses are found to be smaller than those corresponding to the former global test. This contradiction also suggests that two theoretically equivalent set of hypotheses can be rejected at different thresholds, in function of the number and the form of the restrictions used to characterize them. This question has been addressed by several researchers (see e.g. Phillips and Park [1988]). However, the issue on how to best formulate a Wald test for a given set of nonlinear restrictions in empirical research is mainly unsolved.

\subsection{Second Model}

In the second model, the parameter vector $\left(\alpha_{n}^{\top}, \boldsymbol{\alpha}^{\top}, \gamma_{1 n}, \gamma_{2 n}\right)^{\top}$ is estimated from the system of five regressions (49), which is now obtained from (33), (34), (35) and (44). The complete model comprises the 13 additional free parameters $\boldsymbol{\alpha}$. For obtaining the optimal input demand functions $z^{*}$ through Shephard's Lemma, the dependence of the aggregate wage $\mathbf{W}$ on the elementary wages $w$ must be taken into account.

For this extended model, several local maxima were detected and convergence was often very difficult to obtain (an important number of iterations is required, convergence is strongly dependent upon the choice of the starting values). This might be related to the high colinearity between the elementary and the aggregate variables, which both appear as regressors in (44). In the sequel, the estimates corresponding to the highest log-likelihood are retained. The log-likelihood of this extended model is significantly higher than the one of the previous model. ${ }^{8}$

The different elasticity estimates are reported in Table 3. Although Model 1 is rejected

\footnotetext{
${ }^{8}$ This extended input demand system nests the former one, which is obtained when $\boldsymbol{\alpha}=0$. A log-likelihood test rejects this assumption at the one percent level: $2(4012.10-3785.10)=454.0$
} 
against Model 2 at any reasonable significance level, a comparison of the estimates with those reported in Table 2 shows that only few results are quantitatively different. There are only two contradictions between both models, in the sense that an insignificant estimate becomes significant or conversely. Both estimates $\epsilon\left(\ell_{h}^{*}, w_{h}\right)$ and $\epsilon\left(\ell_{u}^{*}, y_{x}\right)$ seem to be economically more plausible in Model 2.

As in the previous subsection, it is possible to test whether the different aggregation conditions of Section 2 are satisfied. The corresponding results for local separability are reported in Table 4. Given the results of Proposition 5, one expects that the separability tests are no longer joint tests for separability and functional form adequacy, and that therefore the separability restrictions should be less often rejected than it was the case with the first model. Nonetheless, the outcome of the tests for the different functional structures are relatively similar to those obtained for the separability inflexible cost function. The mean Wald statistics reported in Table 4 are all relatively similar to those of Table 2. However, the percentage of observations for which the null hypothesis is rejected is in Table 4 never smaller than in Table 2. Only for the test of identical outputs are both percentages the same. This evidence suggests that the strong rejection of the null hypotheses obtained with Model 1, is not due to the loss of flexibility under the null of separability.

As in the first model, output identity is less strongly rejected than the more restrictive functional structures. The test of this assumption yielded the smallest mean Wald statistic (18.4) which leads to less observations than any other functional structure. The persistence of this paradoxical result shows that it was not just a consequence of choosing a more restricted functional form.

For the purpose of comparison, I also computed the Diewert and Wales type of separability tests. The values of the Wald test for restrictions (47), (48), and for both (47) and (48) are respectively 69.6, 1554.5 and 1663.7, leading in all cases to reject the null. As expected, these Wald-test statistics are greater than the mean Wald statistic reported in Tables 2 and 4, due partly to the fact that the validity of the functional restriction is now tested globally (over all observations) and not locally (at each observation point in turn).

The global validity of some functional structures is also tested via the procedure given in Appendix B. Surprisingly, the (nonreported) Wald test for the global validity of the functional structure is then much higher than the parametric test of the corresponding restrictions ((47) and (48)). This additional paradox should also motivate further research on the empirical performance of Wald tests. The above results seem to suggest that the Wald statistic for a given assumption increases, when the assumption is formulated with a greater number of equality restrictions. Unfortunately, tests based on the likelihood or on the regression residuals seem not to be easily implementable in this context. As the restrictions to be tested are nonparametric (even in parametric models), there is no obvious relationship between the restrictions and model' parameters. 
Table 3: Elasticities of Model $2^{(1)}$

\begin{tabular}{ccccccc}
\hline \hline$\epsilon\left(j^{*}, i\right)$ & \multicolumn{1}{c}{$\ell_{h}$} & $\ell_{s}$ & \multicolumn{1}{c}{$\ell_{u}$} & $v_{k}$ & $v_{m}$ \\
\hline$w_{h}$ & -0.26 & 0.06 & -0.10 & -0.02 & 0.01 \\
& $(-4.7)$ & $(5.7)$ & $(-4.9)$ & $(-3.7)$ & $(5.3)$ \\
$w_{s}$ & 0.79 & -0.49 & 0.16 & -0.08 & 0.08 \\
& $(6.0)$ & $(-8.4)$ & $(2.6)$ & $(-3.7)$ & $(7.7)$ \\
$w_{u}$ & -0.95 & 0.09 & -0.40 & -0.02 & 0.05 \\
& $(-5.4)$ & $(2.4)$ & $(-3.1)$ & $(-0.6)$ & $(4.5)$ \\
$q_{k}$ & -0.16 & -0.05 & -0.02 & -0.14 & 0.02 \\
& $(-3.3)$ & $(-2.9)$ & $(-0.8)$ & $(-3.6)$ & $(2.5)$ \\
$q_{m}$ & 0.59 & 0.36 & 0.36 & 0.27 & -0.17 \\
& $(6.9)$ & $(6.5)$ & $(5.2)$ & $(4.4)$ & $(-6.4)$ \\
\hline \multirow{2}{*}{$y_{d}$} & 0.75 & 0.58 & 0.70 & 0.56 & 0.70 \\
& $(15.7)$ & $(7.1)$ & $(11.3)$ & $(11.4)$ & $(10.7)$ \\
$y_{x}$ & 0.23 & 0.08 & 0.09 & 0.15 & 0.15 \\
& $(6.1)$ & $(5.5)$ & $(7.3)$ & $(10.0)$ & $(10.6)$ \\
$t$ & 0.033 & 0.004 & -0.033 & -0.002 & 0.003 \\
& $(11.3)$ & $(3.1)$ & $(-14.9)$ & $(-0.9)$ & $(3.3)$ \\
\hline \hline
\end{tabular}

(1) Median value of the elasticities evaluated at the 1985 data and estimated t-value in parentheses.

Table 4: Tests for functional structure, Model 2

\begin{tabular}{|c|c|c|c|c|}
\hline & $\begin{array}{l}\text { Tested } \\
\text { restrictions }\end{array}$ & $\begin{array}{l}\text { Independent } \\
\text { restrictions }\end{array}$ & $\begin{array}{l}\text { Mean } \\
\text { Wald test }^{(1)}\end{array}$ & $\begin{array}{l}\% \text { obs. with } \\
\mathrm{H}_{0} \text { rejected }^{(2)}\end{array}$ \\
\hline \multicolumn{5}{|l|}{ Outputs: } \\
\hline non-jointness & $(12)$ & 1 & $34.2(19.7)$ & 90.0 \\
\hline separability & (17) & 6 & $35.7(10.3)$ & 93.4 \\
\hline homothetic separability & $(17),(18)$ & 7 & $45.1(22.3)$ & 95.4 \\
\hline additive separability & $(17),(20)$ & 7 & $36.8(9.3)$ & 97.8 \\
\hline hom. add. separability & $(17),(21)$ & 8 & $137.4(68.0)$ & 99.6 \\
\hline identical outputs & $(22)$ & 1 & $18.4(23.2)$ & 62.5 \\
\hline \multicolumn{5}{|l|}{ Labour inputs: } \\
\hline non-jointness & $(14)$ & 3 & $44.6(13.2)$ & 100 \\
\hline separability & $(31)$ & 8 & $61.7(27.4)$ & 98.5 \\
\hline homothetic separability & $(28)$ & 10 & $367.0(176.4)$ & 100 \\
\hline additive separability & $(31),(32)$ & 10 & $65.1(27.0)$ & 98.7 \\
\hline
\end{tabular}

(1) Mean value of the Wald test and calculated standart deviation in parentheses.

(2) There are 456 observations at which the Wald test for a given null hypothesis is calculated. 


\section{Conclusion}

The necessary and sufficient conditions for aggregating (fixed) outputs and (flexible) labour inputs have been derived and used for testing the empirical validity of several exact aggregate representations. The homothetic separability assumption has been relaxed to allow for aggregates that are not homogeneous. The separability assumption has also been strengthen to allow for aggregates to be additive as are those usually provided by statistical offices.

The empirical findings on the possibility to provide an exact aggregate representation of the technology are mainly pessimistic. Although there is some evidence for the local validity of some restrictions, they are globally rejected. The impacts of wages, prices, outputs and time on the different qualifications of labour are too different for allowing aggregation of the three types of labour into a scalar measure. Similarly, domestic and exported outputs are found to be rather different commodities, each being produced from different technological requirements.

A more optimistic conclusion can, however, be drawn from the frequent rejection of restrictions on functional structures. The fact that exact aggregation is statistically invalidated does not necessarily mean that aggregation cannot be achieved, but might be interpreted as evidence for exact aggregation to be too demanding, and that its requirements should be weaken. This is the way followed by the approximate approach, developed by Lewbel [1996] and by Koebel [2002]. In a companion paper, Koebel and Laisney [2005], we illustrate how approximate aggregation can be achieved, using the same data as here for better illustrating the difference between both approaches.

\section{Appendix A: Proof of the Propositions}

Proof of Proposition 1. Homothetic separability $\Rightarrow(17)$ and (18). As homothetic separability in $y$ implies separability in $y$, it is clear that (17) must hold. As $g_{y}$ is homothetic, it can be written as $g_{y}(y)=H(h(y))$ where $h$ is linearly homogeneous. Thus

$$
\frac{\partial g_{y}}{\partial y_{d}} y_{d}+\frac{\partial g_{y}}{\partial y_{x}} y_{x}=H^{\prime} \frac{\partial h(y)}{\partial y_{d}} y_{d}+H^{\prime} \frac{\partial h(y)}{\partial y_{x}} y_{x}=H^{\prime} h(y) .
$$

Using that expression and the fact that $c$ is separable in $y$, that is

$$
c(w, q, y, t)=B_{y}\left(w, q, g_{y}(y), t\right),
$$

leads to

$$
\frac{\partial c}{\partial y_{d}} y_{d}+\frac{\partial c}{\partial y_{x}} y_{x}=\frac{\partial B_{y}}{\partial \mathbf{Y}} H^{\prime} h(y)
$$

with $\mathbf{Y}=g_{y}(y)$. Differentiating (52) with respect to $y_{d}$ gives

$$
\begin{aligned}
& \frac{\partial^{2} c}{\partial y_{d}^{2}} y_{d}+\frac{\partial c}{\partial y_{d}}+\frac{\partial^{2} c}{\partial y_{d} \partial y_{x}} y_{x}=\frac{\partial^{2} B_{y}}{\partial \mathbf{Y}^{2}}\left(H^{\prime}\right)^{2} h(y) \frac{\partial h}{\partial y_{d}}+\frac{\partial B_{y}}{\partial \mathbf{Y}} H^{\prime \prime} \frac{\partial h}{\partial y_{d}} h(y)+\frac{\partial B_{y}}{\partial \mathbf{Y}} H^{\prime} \frac{\partial h}{\partial y_{d}} \\
\Leftrightarrow & \frac{\partial^{2} c}{\partial y_{d}^{2}} y_{d}+\frac{\partial^{2} c}{\partial y_{d} \partial y_{x}} y_{x}=\frac{\partial^{2} B_{y}}{\partial \mathbf{Y}^{2}}\left(H^{\prime}\right)^{2} h(y) \frac{\partial h}{\partial y_{d}}+\frac{\partial B_{y}}{\partial \mathbf{Y}} H^{\prime \prime} \frac{\partial h}{\partial y_{d}} h(y),
\end{aligned}
$$

where the last line is obtained using $\partial c / \partial y_{d}=\left(\partial B_{y} / \partial \mathbf{Y}\right)\left(\partial g_{y} / \partial y_{d}\right)$. As this last expression 
is positive, it is possible to normalize (53) by $\partial c / \partial y_{d}$ for obtaining

$$
\frac{\partial^{2} c}{\partial y_{d}^{2}} \frac{y_{d}}{\partial c / \partial y_{d}}+\frac{\partial^{2} c}{\partial y_{d} \partial y_{x}} \frac{y_{x}}{\partial c / \partial y_{d}}=\left(\frac{\partial^{2} B_{y} / \partial \mathbf{Y}^{2}}{\partial B_{y} / \partial \mathbf{Y}} H^{\prime}+\frac{H^{\prime \prime}}{H^{\prime}}\right) h(y) .
$$

Similarly, differentiating (52) with respect to $y_{x}$ leads to

hence (18).

$$
\frac{\partial^{2} c}{\partial y_{d} \partial y_{x}} \frac{y_{d}}{\partial c / \partial y_{x}}+\frac{\partial^{2} c}{\partial y_{x}^{2}} \frac{y_{x}}{\partial c / \partial y_{x}}=\left(\frac{\partial^{2} B_{y} / \partial \mathbf{Y}^{2}}{\partial B_{y} / \partial \mathbf{Y}} H^{\prime}+\frac{H^{\prime \prime}}{H^{\prime}}\right) h(y),
$$

Conversely, it is well known that (17) implies separability. It remains to show that (18) implies homogeneity. ${ }^{9}$ As $c$ is separable in $y$, the equality

$$
\sum_{o=d, x} \frac{y_{o}}{\partial c / \partial y_{d}} \frac{\partial^{2} c}{\partial y_{d} \partial y_{o}}=\sum_{o=d, x} \frac{y_{o}}{\partial c / \partial y_{x}} \frac{\partial^{2} c}{\partial y_{x} \partial y_{o}}
$$

becomes

$$
\begin{aligned}
& \frac{\frac{\partial^{2} B_{y}}{\partial \mathbf{Y}^{2}}\left(\frac{\partial g_{y}}{\partial y_{d}}\right)^{2}+\frac{\partial B_{y}}{\partial \mathbf{Y}} \frac{\partial^{2} g_{y}}{\partial y_{d} \partial y_{d}}}{\left(\partial B_{y} / \partial \mathbf{Y}\right)\left(\partial g_{y} / \partial y_{d}\right)} y_{d}+\frac{\frac{\partial^{2} B_{y}}{\partial \mathbf{Y}^{2}} \frac{\partial g_{y}}{\partial y_{d}} \frac{\partial g_{y}}{\partial y_{x}}+\frac{\partial B_{y}}{\partial \mathbf{Y}} \frac{\partial^{2} g_{y}}{\partial y_{d} \partial y_{x}}}{\left(\partial B_{y} / \partial \mathbf{Y}\right)\left(\partial g_{y} / \partial y_{d}\right)} y_{x} \\
= & \frac{\frac{\partial^{2} B_{y}}{\partial \mathbf{Y}^{2}} \frac{\partial g_{y}}{\partial y_{d}} \frac{\partial g_{y}}{\partial y_{x}}+\frac{\partial B_{y}}{\partial \mathbf{Y}} \frac{\partial^{2} g_{y}}{\partial y_{d} \partial y_{x}}}{\left(\partial B_{y} / \partial \mathbf{Y}\right)\left(\partial g_{y} / \partial y_{x}\right)} y_{d}+\frac{\frac{\partial^{2} B_{y}}{\partial \mathbf{Y}^{2}}\left(\frac{\partial g_{y}}{\partial y_{x}}\right)^{2}+\frac{\partial B_{y}}{\partial \mathbf{Y}} \frac{\partial^{2} g_{y}}{\partial y_{x} \partial y_{x}}}{\left(\partial B_{y} / \partial \mathbf{Y}\right)\left(\partial g_{y} / \partial y_{x}\right)} y_{x},
\end{aligned}
$$

which can be simplified into

$$
\begin{aligned}
& \frac{\partial^{2} g_{y}}{\partial y_{d} \partial y_{d}} \frac{\partial g_{y}}{\partial y_{x}} y_{d}+\frac{\partial^{2} g_{y}}{\partial y_{d} \partial y_{x}} \frac{\partial g_{y}}{\partial y_{x}} y_{x}=\frac{\partial^{2} g_{y}}{\partial y_{d} \partial y_{x}} \frac{\partial g_{y}}{\partial y_{d}} y_{d}+\frac{\partial^{2} g_{y}}{\partial y_{x} \partial y_{x}} \frac{\partial g_{y}}{\partial y_{d}} y_{x} \\
\Leftrightarrow & \left(\frac{\partial^{2} g_{y}}{\partial y_{d} \partial y_{d}} \frac{\partial^{2} g_{y}}{\partial g_{y} / \partial y_{d}}-\frac{\partial^{2} g_{y} \partial y_{x}}{\partial g_{y} / \partial y_{x}}\right) y_{d}=\left(\frac{\frac{\partial^{2} g_{y}}{\partial y_{x} \partial y_{x}}}{\partial g_{y} / \partial y_{x}}-\frac{\frac{\partial y_{d} \partial y_{x}}{\partial g_{y} / \partial y_{d}}}{y_{x}}\right) y_{x} \\
\Leftrightarrow & \frac{\partial \ln \left(\frac{\partial g_{y} / \partial y_{d}}{\partial g_{y} / \partial y_{x}}\right)}{\partial y_{d}} y_{d}=\frac{\partial \ln \left(\frac{\partial g_{y} / \partial y_{x}}{\partial g_{y} / \partial y_{d}}\right)}{\partial y_{x}} y_{x} .
\end{aligned}
$$

As a consequence, the function $\left(\partial g_{y} / \partial y_{d}\right) /\left(\partial g_{y} / \partial y_{x}\right)$ is homogeneous of degree zero in $y$, and therefore $g_{y}$ is homothetic by Lau's [1970] Lemma 1.

Proof of proposition 2. The condition

$$
c\left(w, q, y_{d}, y_{x}, t\right)=B_{y}\left(w, q, \delta_{d} y_{d}+\delta_{x} y_{x}, t\right),
$$

is equivalent to

which in turn is equivalent to (17) and (21).

$$
\frac{\partial c / \partial y_{d}}{\partial c / \partial y_{x}}=\frac{\partial g_{y} / \partial y_{d}}{\partial g_{y} / \partial y_{x}}=\frac{\delta_{d}}{\delta_{x}},
$$

Lemma 1. There exist two continuously differentiable real valued function $g_{\ell}$ and

\footnotetext{
${ }^{9}$ That (18) alone does not imply separability should be clear from the following example. The cost function

$$
c(w, q, y, t)=c_{1}(w, q, t) \sqrt{y_{d}}+c_{2}(w, q, t) \sqrt{y_{x}}
$$
}

satisfies (18), but is not separable in $y$ as long as $c_{1}$ and $c_{2}$ are not proportional. 
$A_{\ell}$, such that

$$
a(\ell, q, y, t) \equiv \min _{v}\left\{q^{\top} v: f(\ell, v, y, t)=0\right\}=A_{\ell}\left(g_{\ell}(\ell), q, y, t\right)
$$

if and only if

$$
\operatorname{rank}\left[\frac{\partial^{2} a}{\partial \ell \partial\left(q^{\top}, y^{\top}, t\right)}\right] \leq 1
$$

Proof of Lemma 1. Let $\mathbf{L}=g_{\ell}(\ell)$, then condition (55) implies that

$$
\frac{\partial^{2} a}{\partial \ell \partial\left(q^{\top}, y^{\top}, t\right)}=\partial\left(\frac{\partial A_{\ell}}{\partial \mathbf{L}} \frac{\partial g_{\ell}}{\partial \ell}\right) / \partial\left(q^{\top}, y^{\top}, t\right)=\frac{\partial^{2} A_{\ell}}{\partial \mathbf{L} \partial\left(q^{\top}, y^{\top}, t\right)} \frac{\partial g_{\ell}}{\partial \ell},
$$

whose rank is clearly one or zero. Conversely, (56) means that any $2 \times 2$ submatrix of $\partial^{2} a / \partial \ell \partial\left(q^{\top}, y^{\top}, t\right)$ has zero determinant, in which case

$$
\left(\frac{\partial a / \partial \ell_{i}}{\partial a / \partial \ell_{j}}\right) / \partial\left(q^{\top}, y^{\top}, t\right)=0
$$

implying, as shown by Leontief [1947], the existence of a function $g_{\ell}$ such that (55) holds.

A similar Lemma can be derived to give the conditions on $f$ that are equivalent to $f(\ell, v, y, t)=G_{\ell}\left(g_{\ell}(\ell), v, y, t\right)$.

Proof of proposition 3. Different and much longer proofs of a related result have been given by Blackorby, Davidson and Schworm [1991] and by Koebel [2001]. Since under cost minimization,

$$
f(\ell, v, y, t)=G_{\ell}\left(g_{\ell}(\ell), v, y, t\right) \Leftrightarrow a(\ell, q, y, t)=A_{\ell}\left(g_{\ell}(\ell), q, y, t\right),
$$

the proof of the left hand equality can rely on the right hand equality. The first order conditions

$$
\frac{\partial a}{\partial \ell}\left(\ell^{*}(w, q, y, t), q, y, t\right)=w
$$

corresponding to minimization of $a$ with respect to $\ell$, together with

$$
c(w, q, y, t)=w^{\top} \ell^{*}(w, q, y, t)+a\left(\ell^{*}(w, q, y, t), q, y, t\right),
$$

lead to

$$
\begin{aligned}
\frac{\partial c}{\partial w} & =\ell^{*}(w, q, y, t) \\
\frac{\partial^{2} a}{\partial \ell \partial \ell^{\top}} \frac{\partial^{2} c}{\partial w \partial w^{\top}} & =I_{d}
\end{aligned}
$$

where $I_{d}$ denote the identity matrix. From this last line it can be seen that $\partial^{2} a / \partial \ell \partial \ell^{\top}$ is the inverse of $\partial^{2} c / \partial w \partial w^{\top}$. Differentiation of $\partial a / \partial \ell$ with respect to $\left(q^{\top}, y^{\top}, t\right)$ then yields

$$
\begin{aligned}
\frac{\partial^{2} a}{\partial \ell \partial \ell^{\top}} \frac{\partial^{2} c}{\partial w \partial\left(q^{\top}, y^{\top}, t\right)} & =-\frac{\partial^{2} a}{\partial \ell \partial\left(q^{\top}, y^{\top}, t\right)} \\
\frac{\partial^{2} c}{\partial w \partial\left(q^{\top}, y^{\top}, t\right)} & =-\left[\frac{\partial^{2} a}{\partial \ell \partial \ell^{\top}}\right]^{-1} \frac{\partial^{2} a}{\partial \ell \partial\left(q^{\top}, y^{\top}, t\right)} .
\end{aligned}
$$

$(i) \Leftrightarrow(i i)$ : By $(61) \operatorname{rank} \partial^{2} c / \partial w \partial\left(q^{\top}, y^{\top}, t\right)=\operatorname{rank} \partial^{2} a / \partial \ell \partial\left(q^{\top}, y^{\top}, t\right)$. The conclusion follows from Lemma 1. 
(ii) $\Leftrightarrow$ (iii) directly follows from the definition of the elasticities.

Proof of proposition 4. As there are 3 types of labour inputs, the Leontief conditions for additive separability in $\ell$ can be applied, stating that $a$ is additive in $\ell$ iff (30) is satisfied and

$$
\partial\left(\frac{\partial a / \partial \ell_{h}}{\partial a / \partial \ell_{s}}\right) / \partial \ell_{u}=\partial\left(\frac{\partial a / \partial \ell_{s}}{\partial a / \partial \ell_{u}}\right) / \partial \ell_{h}=\partial\left(\frac{\partial a / \partial \ell_{u}}{\partial a / \partial \ell_{h}}\right) / \partial \ell_{s}=0,
$$

or equivalently ${ }^{10}$

$$
\begin{aligned}
\frac{1}{w_{h}} \frac{\partial^{2} a}{\partial \ell_{h} \partial \ell_{u}} & =\frac{1}{w_{s}} \frac{\partial^{2} a}{\partial \ell_{s} \partial \ell_{u}} \\
\frac{1}{w_{s}} \frac{\partial^{2} a}{\partial \ell_{h} \partial \ell_{s}} & =\frac{1}{w_{u}} \frac{\partial^{2} a}{\partial \ell_{h} \partial \ell_{u}} .
\end{aligned}
$$

The conditions (30), (63) can equivalently be given in terms of function $c$. From Proposition $3,(30)$ is equivalent to (31). From (59), and the formula of the inverse of a matrix, restrictions (63) become

$$
\begin{gathered}
\frac{1}{w_{h}} \operatorname{det}\left[\begin{array}{cc}
\frac{\partial^{2} c}{\partial w_{h} \partial w_{s}} & \frac{\partial^{2} c}{\partial w_{s} \partial w_{s}} \\
\frac{\partial^{2} c}{\partial w_{h} \partial w_{u}} & \frac{\partial^{2} c}{\partial w_{s} \partial w_{u}}
\end{array}\right]=-\frac{1}{w_{s}} \operatorname{det}\left[\begin{array}{cc}
\frac{\partial^{2} c}{\partial w_{h} \partial w_{h}} & \frac{\partial^{2} c}{\partial w_{h} \partial w_{s}} \\
\frac{\partial^{2} c}{\partial w_{h} \partial w_{u}} & \frac{\partial^{2} c}{\partial w_{s} \partial w_{u}}
\end{array}\right] \\
-\frac{1}{w_{s}} \operatorname{det}\left[\begin{array}{cc}
\frac{\partial^{2} c}{\partial w_{h} \partial w_{s}} & \frac{\partial^{2} c}{\partial w_{s} \partial w_{u}} \\
\frac{\partial^{2} c}{\partial w_{h} \partial w_{u}} & \frac{\partial^{2} c}{\partial w_{u} \partial w_{u}}
\end{array}\right]=\frac{1}{w_{u}} \operatorname{det}\left[\begin{array}{cc}
\frac{\partial^{2} c}{\partial w_{h} \partial w_{s}} & \frac{\partial^{2} c}{\partial w_{s} \partial w_{s}} \\
\frac{\partial^{2} c}{\partial w_{h} \partial w_{u}} & \frac{\partial^{2} c}{\partial w_{s} \partial w_{u}}
\end{array}\right],
\end{gathered}
$$

which leads to (32).

Proof of Proposition 5: Diewert and Wales [1995, Proposition 2] prove point (i) for the normalized quadratic functional form. Adapting their proof to the somewhat different context of the Box-Cox function shows that $c$ is flexible in $(w, q, y, t)$. Point (ii): Set

$$
B_{Q Y}=0, B_{W Y}=0, B_{Y t}=0, \mathbf{B}_{\mathbf{W} Y}=0,
$$

in the expression of $\mathbf{C}_{\mathbf{W Y}}$. Then, it is direct to see that $c$ is separable in $y$. Moreover, under (64), $\mathbf{C}_{\mathbf{W Y}}$ is still quadratic in $(W, Q, \mathbf{Y}, t)$. Thus the first and second order derivatives of $\mathbf{C}_{\mathbf{W Y}}$ can locally approximate those of an arbitrary function of $(w, q, \mathbf{y}, t)$ (hence the local flexibility). Point (iii) and (iv) are obtained similarly.

\section{Appendix B: A global test}

For testing the overall validity of the different null hypotheses, one first needs to stack up the local test statistics $\widehat{s}_{n t} \equiv s_{n t}\left(p_{n t}, y_{n t}, t, \widehat{\alpha}_{n}\right)$ overall $n$ and $t$ to obtain $\widehat{s} \equiv$ $\left(\widehat{s}_{11}, \ldots, \widehat{s}_{N T}\right)^{\top}$. In the local tests reported in Tables 2 and 4 , the hypothesis $s_{n t}\left(\alpha_{n}\right)=0$ (with an abuse of notation for $s_{n t}\left(p_{n t}, y_{n t}, t, \alpha_{n}\right)=0$ ) is tested for any $n$ and $t$. Asymptotically $\widehat{s}_{n t} \stackrel{a}{\sim} N\left(s_{n t},\left(\partial s_{n t}^{\top} / \partial \alpha_{n}\right) \Omega_{n}\left(\partial s_{n t} / \partial \alpha_{n}^{\top}\right)\right)$, with $\mathrm{V}\left(\alpha_{n}\right)=\Omega_{n}$. However, comput-

\footnotetext{
${ }^{10}$ One of the three equalities is redondant.
} 
ing the variance of $\widehat{s}_{n t}$ using the delta method is a relatively time consuming task. ${ }^{11}$ For testing whether $s(\alpha) \equiv\left(s_{11}\left(\alpha_{1}\right), \ldots, s_{N T}\left(\alpha_{N}\right)\right)^{\top}=0$, an estimate $\widehat{\Upsilon}$ of the variance matrix $\Upsilon$ of $\widehat{s}$ is required. In the present case, the dimension of the matrix $\Upsilon$ is comprised between $(456 \times 456)$ and $(4560 \times 4560)$, depending upon the functional structure being tested. Approximating the matrix $\Upsilon$ by using the delta method (yielding $\left.\Upsilon_{\Delta}=\left(\partial s^{\top} / \partial \alpha\right) \Omega\left(\partial s / \partial \alpha^{\top}\right)\right)$ would require months of calculation. To avoid that drawback, I rely instead on a resampling method for obtaining an estimate of $\Upsilon$.

As $D\left(\widehat{\alpha}_{N T}-\alpha\right) \stackrel{d}{\rightarrow} \mathcal{N}(0, \Omega)$ where $\Omega$ is finite and positive definite and $D$ is a diagonal matrix of scaling factors (depending upon $N$ and $T$ ), it follows that $\mathcal{N}(D \alpha, \Omega)$ is the limiting density of $\left\{D \widehat{\alpha}_{N T}, D \widehat{\Omega}_{N T} D\right\}$, where $\widehat{\Omega}_{N T}$ is the estimated variance matrix of $\widehat{\alpha}_{N T}$. Thus, the density $\mathcal{N}\left(\widehat{\alpha}_{N T}, \widehat{\Omega}_{N T}\right)$ can be used to draw a sample of $\widehat{\alpha}^{i}, i=1, \ldots, I$, for which the value of $s_{n t}^{i}\left(p_{n t}, y_{n t}, t, \widehat{\alpha}_{n}^{i}\right) \equiv \widehat{s}_{n t}^{i}$ is calculated for each observation point $n$ and $t$. The vector $\widehat{s}^{i}$ is obtained by stacking up $\widehat{s}_{n t}^{i}$ over $n$ and $t$. Then, it is easy to calculate the empirical mean $\bar{s}_{I}=I^{-1} \sum_{i} \widehat{s}^{i}$ and variance matrix $\widehat{\Upsilon}_{I}=I^{-1} \sum_{i}\left(\widehat{s}^{i}-\bar{s}_{I}\right)\left(\widehat{s}^{i}-\bar{s}_{I}\right)^{\top}$ of $\widehat{s}^{i}$. By the law of large numbers, this matrix $\widehat{\Upsilon}_{I}$ converges in probability to $\Upsilon$. These are the steps I rely on to calculate the Wald statistic $\widehat{W}=\widehat{s}^{\top} \widehat{\Upsilon}_{I}^{-} \widehat{s}$, for testing the (global) null hypothesis $s=0$. In my experimentation I chose to set $I$ either to 10000 or to 30000, values for which the outcomes of the Wald test were no longer changing very much with increasing $I$.

A comparison of the estimates of the diagonal terms of $\Upsilon_{\Delta}$ (the variances obtained using the delta method and used in the local test of functional structure) with those of $\widehat{\Upsilon}_{I}$ reveals a relatively small difference of about five percent in average. However, for some few observations the gap is about $50 \%$ between the two estimates of the variance. As for $I$ high enough, $\widehat{\Upsilon}_{I}$ provides a better approximation to $\Upsilon$ than $\widehat{\Upsilon}_{\Delta}$, I have some preference for the results based on $\widehat{\Upsilon}_{I}$. The percentage of observations for which the null is rejected is, however, very similar independently of the method relied on.

\section{References}

Baumol W. J., J. C. Panzar and R. D. Willig, 1982, Contestable Markets and the Theory of Industry Structure, New York: Harcourt Brace Jovanovich Inc.

Berndt, E. R., and L. R., Christensen, 1973, 'The Internal Structure of Functional Relationships: Separability and Aggregation', Review of Economic Studies, 40, pp.403-410.

Berndt, E. R., and L. R., Christensen, 1974, 'Testing for the Existence of a Consistent Aggregate Index of Labor Inputs', American Economic Review, 64, pp.391-404.

Berndt, E. R. and M. S. Khaled, 1979, 'Parametric productivity measurement and choice among flexible functional forms', Journal of Political Economy, 87, 1220-1245.

Berndt, E. R., and N. E. Savin, 1975, 'Estimation and Hypothesis Testing in Singular Equation Systems with Autoregressive Disturbances', Econometrica, 43, pp.937-957.

Blackorby, C., and W. E. Diewert, 1979, 'Expenditure Functions, Local Duality, and Second Order Approximations', Econometrica, 47, pp. 579-601.

Blackorby, C., and R. R. Russell, 1976, 'Functional Structure and the Allen Partial Elasticities of

\footnotetext{
${ }^{11}$ Up to 500 seconds were necessary for calculating the local test (one observation point), using TSP version 4.5 on a Pentium III personal computer.
} 
Substitution: An Application of Duality Theory', Review of Economic Studies, 43, pp.285-291.

Blackorby, C., and W. Schworm, 1993, 'The Implications of Additive Community Preferences in a Multi-Consumer Economy', Review of Economic Studies, 60, pp.209-227.

Blackorby, C., R. Davidson, and W. Schworm, 1991, 'Implicit Separability: Characterisation and Implications for Consumer Demands', Journal of Economic Theory, 55, pp.364-399.

Blackorby, C., Primont, D. and Russell, R. R., 1977, 'On Testing Separability Restrictions with Flexible Functional Forms', Journal of Econometrics, 5, pp.195-209.

Blackorby, C., Primont, D. and Russell, R. R., 1978, Duality, Separability, and Functional Structure: Theory and Economic Applications, New York: North-Holland.

Blackorby, C., W. Schworm and T. Fisher, 1986, 'Testing the Existence of Input Aggregates in an Economy Production Function', Discussion Paper 86-26 University of British Columbia, Vancouver.

Diewert, W. E., 1974, 'Applications of Duality Theory', in M. D. Intriligator and D. A. Kendrick, Frontiers of Quantitative Economics, Volume 2, North-Holland.

Diewert, W. E. and T. J. Wales, 1987, 'Flexible functional forms and global curvature conditions', Econometrica, 55, pp. 47-68.

Diewert, W. E. and T. J. Wales, 1995, 'Flexible Functional Forms and Tests of Homogeneous Separability', Journal of Econometrics, 67, pp.259-302.

Gorman, W. M., 1995, Separability and Aggregation, Collected Works of W. M. Gorman, Volume I, edited by C. Blackorby and A. F. Shorrocks, Clarendon Press: Oxford, 1995.

Hall, R. E., 1973, 'The specification of technology with several kinds of ouput', Journal of Political Economy, 81, pp.878-892.

Koebel, B. M., 2001, 'Separabilities and Elasticities', Discussion Paper No. 07-2001, Department of Economics, University College London.

Koebel, B. M., 2002, 'Can Aggregation be Achieved by Neglecting the Problem? Property Inheritance and Aggregation Biases', International Economic Review, 43, pp.223-255.

Koebel, B. M., 2004, 'First order serial correlation in seemingly unrelated regressions', Economics Letters 82, 1-7.

Koebel, B. M., 2005, 'Approximate Aggregation in Multi-Output Multi-Skill Technologies', mimeo, Louis Pasteur University, Strasbourg.

Koebel, B. M., M. Falk, and F. Laisney, 2003, 'Imposing and Testing Curvature Conditions on a Box-Cox Cost Function', Journal of Business and Economic Statistics, 21, 319-335.

Kohli, U., 1983, 'Non-joint technologies', Review of Economic Studies, 50, pp.209-219.

Leontief, W., 1947, 'Introduction to a Theory of the Internal Structure of Functional Relationships', Econometrica, 15, pp.361-373.

Phillips, P. C. B., and J. Y. Park, 1988, 'On the Formulation of Wald Tests of Nonlinear Restrictions', Econometrica, 56. pp.1065-1083.

Russell, R. R., 1975, 'Functional Separability and Partial Elasticities of Substitution', Review of Economic Studies, 42, pp.79-85. 